628.53

G229cx

GATZ

CHARACTERIZATION OF URBAN AND RURAL INHALABLE PARTICULATES 
Digitized by the Internet Archive in 2013

http://archive.org/details/characterization8311gatz 


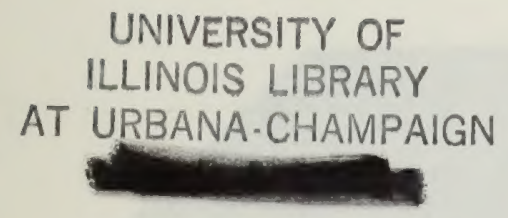





\section{CHARACTERIZATION OF URBAN AND RURAL INHALABLE PARTICULATES}

\section{Document No. 83/11}

\section{DEPOSITORY}

JUL 61983

UNIVERSITY OF ILLINOIS AT URBANA.CHAMPAIGN

Energy and Natural Resources

James R. Thompson, Governor Michael B. Witte, Director 
UNIVERSITY OF ILLINOIS LIBRARY

AT. URBANA-CHAMPAIGN 
ENR Doc. No. $83 / 11$

SWS Contract Report 308

February, 1983

CHARACTERIZATION OF URBAN AND RURAL

INHALABLE PARTICULATES

by

Donald F. Gatz 1)

Susan T. Wiley

Lih-Ching Chu

Project No. 10.093

James R. Thompson, Governor

State of Illinois
Michael B. Witte, Director Department of Energy and Natural Resources

1) Prepared under contract with the IIlinois Department of Energy and Natural Resources as project number 10.093; from the State Water Survey Division, Champaign, Illinois. 
This report has been reviewed by the Department of Energy and Natural Resources and approved for publication by ENR.

Printed by the Authority of the State of Illinois.

\author{
Date Printed: $\quad$ March, 1983 \\ Quantity Printed: 200
}

One of a series of research publications published since 1975. This series includes the following categories and are color coded as follows:

\begin{tabular}{lcll} 
Air Quality & $\begin{array}{c}\text { Prior to } \\
\text { July, 1982 }\end{array}$ & & $\begin{array}{c}\text { After } \\
\text { July, 1982 }\end{array}$ \\
\cline { 2 - 2 } Water & - Green & & Green \\
Environmental Health & - Blue & & Blue \\
Solid and Hazardous Waste & - White & Grey \\
Economic Impact Study & - Buff & Brown \\
Noise Management & - Buff & Orange \\
Energy & - Cherry & Red \\
Information Services & - Canary & Yellow
\end{tabular}

Illinois Department of Energy and Natural Resources Division of Policy and Planning

325 West Adams Street Springfield, Illinois 62706

(217) $785-2800$ 
List of Figures

List of Tables

1 EXECUTIVE SUMMARY

1.1 ISSUES

1.1.1 Impacts and control of fugitive dust sources of TSP

1.1.2 Inhalable mass vs total particle mass concentration

1.2 THE AUDIENCE FOR THESE RESULTS

1.3 OBJECTIVES

1.4 FINDINGS

1.4.1 Comparisons of Inhalable and Total Particle Mass

1.4.2 Effects of Street Sweeping on Urban Aerosol Concentrations

1.4.3 Sources of Urban Aerosols, and their Relative Importance

2 INTRODUCTION

3 LITERATURE REVIEW

3.1 SOURCE IDENTIFICATION AND APPORTIONMENT

3.2 WIND EROSION

3.2.1 Introduction and History

3.2.2 Wind Erosion Processes

3.2.2.1 Natural Wind

3.2.2.2 Saltation

3.2.2.3 Surface Creep

3.2.2.4 Suspension

3.2.2.5 Effects on the Soil

3.2.3 Factors Affecting Wind Erosion

3.2.3.1 Soil Cloddiness

3.2.3.2 Surface Roughness

3.2.3.3 Wind Speed and Soil Moisture 21

3.2.3.4 Unsheltered Field Length 22

3.2.3.5 Vegetative Cover 23

3.2.4 Wind Erosion Equation 24

3.2 .5 Summary 26 
$\begin{array}{ll}3.3 & \text { EMISSIONS FROM PAVED AND UNPAVED ROADS }\end{array}$

3.4 COMPARISON OF AEROSOL MEASUREMENT METHODS 28

4 EXPERIMENTAL METHODS 28

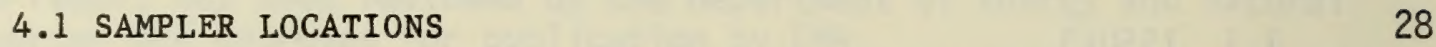

\begin{tabular}{ll}
4.2 & SAMPLING METHODS \\
\hline
\end{tabular}

4.3 ANALYSIS METHODS 32

4.4 STREET SWEEPING OPERATIONS 33

4.5 SUPPORTING DATA 34

4.6 SOURCE APPORTIONMENT BY CHEMICAL ELEMENT BALANCE 35

5 RESULTS AND DISCUSSION 37

5.1 RURAL MEASUREMENTS 37

5.1.1 Comparison of Inhalable and Total Aerosols 37

5.2 URBAN HIVOL MEASUREMENTS 42

5.2.1 Data Summary 42

5.2.2 Effects of Street Sweeping 44

5.2.3 TSP Concentrations Upwind and Downwind of
Mattis Avenue

5.2.4 TSP Concentrations in Commercial and
Residential Areas with and without Sweeping

5.3 URBAN SOURCE APPORTIONMENT USING DICHOTOMOUS SAMPLER
MEASUREMENTS

5.3.1 The Data 56

5.3.2 CEB on Mean Ambient Concentrations 64

5.3.3 CEBs on Individual Samples 86

6 SUMMARY AND CONCLUSIONS 96

7 ACKNOWLEDGEMENTS 98

8 REFERENCES 100 
Number

Figure 1. Map of a portion of Champaign, Illinois,

showing aerosol sampling sites, drainage basins, and sampling sites of the Water Survey's project in the Nationwide Urban Runoff Program.

Figure 2. Comparison of rural particle mass concentrations measured by standard and size-selective high volume samplers.

Figure 3. Illustration of data collected in 1981 for evaluation of effects of street sweeping on air quality.

Figure 4. Comparison of TSP concentrations upwind and downwind of Mattis Avenue on days when winds were predominantly from the east or west.

Figure 5. Comparison of TSP concentrations on the east and west sides of Mattis Avenue on days when winds were not predominantly from the east or west.

Figure 6. Summary of source contributions to total aerosol mass.

Figure 7. Frequency distributions of source contributions, based on CEB analyses of individual samples -- fine aerosol.

Figure 8. Frequency distributions of source contributions, based on CEB analyses of individual samples -- coarse aerosol.

Figure 9. Mean street dust contributions to total mass $<15$ m as a function of wind direction.

Figure 10. Street dust contribution to total mass $<15$ $m$ as a function of the fraction of time winds were from the westerly sector during sample collection. 
Table 1. Comparison of inhalable particle/total

particle ratios in this study with literature values.

Table 2. Results of 2-way analys is of variance on upwind-downwind TSP differences across the test roadway.

Table 3. Results of 2-way analysis of variance of commercial-residential TSP differences.

Table 4. Summary of TSP concentrations and differences by season for three different sample sets.

Table 5. Summary of ambient fine particle elemental concentrations measured on 95 sampling days, Mattis Ave., Champaign, Illinois, June October, 1981 .

Table 6. Summary of ambient coarse particle elemental concentrations measured on 95 sampling days, Mat t.is Ave., Champaign, Illinois, June October, 1981 .

Table 7. Source compositions (\%), fine fraction, based on resuspended samples.

Table 8. Source compositions (\%), coarse fraction, based on resuspended samples.

Table 9. Summary of CEB results on fine particles, Champaign dichotomous filter data, based on mean observed element concentrations.

Table 10. Chemical element balance for Champaign dichotomous fine particles.

Table 11. Summary of CEB results on coarse particles, Champaign dichotomous filter data, based on mean observed element concentrations.

Table 12. Chemical element balance for Champaign dichotomous coarse particles.

Table 13. Summary of major sources and fit characteristics of individual elements in fine and coarse particle classes. 
1 EXECUTIVE SUMMARY

\subsection{ISSUES}

1.1.1 Impacts and Control of Fugitive Dust Sources of TSP

Provisions of the Clean Air Act enacted in 1977 require states to revise their State Implementation Plans (SIPs) for all areas that have not attained National Ambient Air Quality Standards (NAAQS). The Illinois SIP for Total Suspended Particulates (TSP) was conditionally approved by the U.S. Environmental Protection Agency (USEPA) with certain minor deficiencies. One of the reasons for the conditional approval of the IIlinois TSP SIP was inadequate documentation of the impacts and effects of various controls on non-traditional fugitive sources of TSP. Examples of these sources include reentrained road dust, wind erosion from agricultural lands, and unpaved road emissions. This and similar studies have been designed to correct those deficiencies and will be submitted to the USEPA as part of the SIP for that purpose. The results of these studies will be used by the Illinois Environmental Protection Agency (IEPA) to define further the estimated impacts of non-traditional fugitive dust sources on TSP non-attainment areas throughout the state. They will also be used to refine the 
control strategies which may need to be applied to various non-traditional fugitive dust sources.

1.1.2 Inhalable Mass vs Total Particle Mass Concentrations

The Clean Air Act, as most recently amended (1977), emphasizes that health aspects should be considered very strongly when assessing effects of air pollutants. This has caused a concern that the standard high volume (hivol) sampler does not provide a sample of particles in the limited size range important for assessing health effects. Thus, the standard hivols may soon need to be replaced with samplers that measure concentrations of particles within the specific size range known to be capable of reaching the lungs through inhalation. Several such devices are under consideration. One is the two-stage virtual impactor (dichotomous sampler), which collects particles in two size ranges: less than about $2.5 \mu \mathrm{m}$ and 2.5 to $15 \mu \mathrm{m}$, aerodynamic diameter. Others include size-selective inlets for the standard hivols. These devices limit the particles collected to those smaller than some cutoff diameter, such as $15 \mu \mathrm{m}$. In this report, we shall consider inhalable particles to be those less than or equal to $15 \mu \mathrm{m}$ aerodynamic diameter. 


\subsection{THE AUDIENCE FOR THESE RESULTS}

The results of this study should be of interest to pollution control officials that have responsibility for monitoring ambient pollutant concentrations and those that must plan strategies for meeting air pollution standards. The results should also be of interest to atmospheric chemists interested in sources of urban aerosols and their relative contributions to ambient concentrations of both total mass and individual elements.

\subsection{OBJECTIVES}

The purposes of the study were:

1. To compare concentrations of inhalable and total airborne mass in both urban and rural areas,

2. To assess the effects of street sweeping on urban air quality, by comparing hivol and dichotomous sampler measurements in urban areas in the presence and absence of regular street sweeping, and

3. To determine the sources of airborne particulate matter, and their relative contributions to TSP, in an urban area. 


\subsection{FINDINGS}

1.4.1 Comparisons of Inhalable and Total Particle Mass

Concentrations of inhalable particles in a rural area, as measured by a size-selective hivol sampler, averaged $75 \%$ of those of TSP measured in a standard hivol.

Mean inhalable mass concentrations in Champaign, as measured by a dichotomous sampler, were about $50 \%$ of the mean TSP concentrations measured by a standard hivol.

Thus, IP-TSP comparisons in urban and rural areas of central Illinois agree very closely with similar comparisons in the literature, made in other parts of the country.

1.4.2 Effects of Street Sweeping on Urban Aerosol Concentrations 
Dust from the test roadway increased TSP at $7 \mathrm{~m}$ downwind by an average of about $22 \mu \mathrm{g} / \mathrm{m}^{3}$ with winds within \pm 68 degrees of perpendicular to the roadway. Yet, no evidence could be found that routine weekly sweeping had any effect in reducing (or increasing) aerosol concentrations near the roadway. It appears that the inefficient removal of street particles $<125 \mu \mathrm{m}$ in diameter by street sweepers is crucial, since the particles of street dust that become and remain airborne are in this size range.

1.4.3 Sources of Urban Aerosols, and their Relative Importance

Source apportionment calculations indicated that atmospheric sulfate accounted for more than $50 \%$ of the fine $(<2.5 \mu \mathrm{m})$ aerosol. Street dust accounted for only $2 \%$ of the fine mass, but $66 \%$ of the coarse $(2.5-15$ $\mu \mathrm{m})$ mass, and by inference, about $50 \%$ of hivol TSP concentrations.

The influence of nearby Mattis Ave could be seen in the hivol measurements, but not in those from the dichotomous sampler, so the major contribution of Mattis Ave must have been in particles larger than $15 \mu \mathrm{m}$. 
Provisions of the Clean Air Act enacted in 1977 require states to revise their State Implementation Plans (SIPs) for all areas that have not attained National Arabient Air Quality Standards (NAAQS). The Illinois SIP for Total Suspended Particulates (TSP) was conditionally approved by the U.S. Environmental Protection Agency (USEPA) with certain minor deficiencies. One of the reasons for the conditional approval of the Illinois TSP SIP was inadequate documentation of the impacts and effects of various controls on non-traditional fugitive sources of TSP. Examples of these sources include reentrained road dust, wind erosion from agricultural lands, and unpaved road emissions. This and similar studies have been designed to correct those deficiencies and will be submitted to the USEPA as part of the SIP for that purpose. The results of these studies will be used by the Illinois Environmental Protection Agency (IEPA) to define further the estimated impacts of non-traditional fugitive dust sources on TSP non-attainment areas throughout the state. They will also be used to refine the control strategies which may need to be applied to various non-traditional fugitive dust sources.

The Clean Air Act, as most recently amended (1977), emphasizes that health aspects should be considered very strongly when assessing effects 
of air pollutants. This has caused a concern that the standard high volume (hivol) sampler does not provide a sample of particles in the limited size range important for assessing health effects. Thus the standard hivols may soon need to be replaced with samplers that measure concentrations of particles within the specific size range known to be capable of reaching the lungs through inhalation. Several such devices are under consideration. One is the two-stage virtual impactor (dichotomous sampler), which collects particles in two size ranges: less than about $2.5 \mathrm{~m}$ and 2.5 to $15 \mathrm{~m}$, aerodynamic diameter. Others include size-selective inlets for the standard hivols. These devices limit the particles collected to those smaller than some cutoff diameter, such as $15 \mathrm{~m}$. In this report, we shall consider inhalable particles to be those less than or equal to $15 \mathrm{~m}$ aerodynamic diameter.

In October, 1980, the Illinois Institute of Natural Resources (now the Department of Energy and Natural Resources) initiated a 10-month first phase study with the State Water Survey Division to characterize urban and rural particulate matter. A second phase was funded in November, 1981. This is a final report of results on both phases through 30 June 1982 .

The purposes of the study were:

1. To compare concentrations of inhalable and total airborne mass in both urban and rural areas, 
2. To assess the effects of street sweeping on urban air quality, by comparing hivol and dichotomous sampler measurements in urban areas in the presence and absence of regular street sweeping, and

3. To determine the sources of airborne particulate matter, and their relative contributions to TSP, in an urban area.

This study was planned so as to utilize the regular street sweeping program being conducted in Champaign, Illinois, by a research group from the Water Survey's Surface Water Section and funded by EPA as part of the Nationwide Urban Runoff Program (NURP); the Principal Investigator of this study is Michael L. Terstriep, Head of the Surface Water Section.

\section{LITERATURE REVIEW}

\subsection{SOURCE IDENTIFICATION AND APPORTIONMENT}

Techniques have been developed in recent years whereby measurements of elemental concentrations of ambient aerosols may be analyzed in such a way that the sources of the aerosols may be identified. These identifications may be qualitative, i.e., simply listing the types of sources present, without any indication of their contributions to the total aerosol concentration, or they may be quantitative, giving 
estimates of actual contributions by the various sources. Conventional factor analyses and cluster analyses yield qualitative source identifications, while chemical element balance (CEB) methods and target transformation factor analyses yield quantitative information. These methods of source identification and apportionment have come to be known collectively as "receptor models," since they depend on data measured at receptors, rather than sources. Reviews of this field have been provided by Gordon $(1980 a, b)$.

Receptor models, both qualitative and quantitative, commonly find "crustal dust," or a similarly-named source, perhaps "soil dust" or "limestone," to be a source of ambient aerosol. Miller et al, (1972), using a CEB method, found that soil dust contributed about $10 \%$ of the aerosol in Pasadena, California. Using a very similar technique, Gatz (1975) found that soil dust accounted for about 18\% of Chicago aerosols. Using aerosol composition data collected at St. Louis in Project METROMEX, Gatz (1978) qualitatively identified a "soil and flyash" source, using factor analysis. Dzubay (1980) used a CEB method on dichotomous sampler data from the St. Louis Regional Air Pollution Study (RAPS) and found that "shale" and "limestone" accounted for $86 \%$ of the coarse $(2.4-20 \mu \mathrm{m})$ particles, $10 \%$ of the fine $(<2.4 \mu \mathrm{m})$ particles, and $43 \%$ of the total particle mass. Hopke et al, (1976) applied factor analysis to aerosol composition data from the Boston area and identified a "crustal" source, perhaps combined to some extent with flyash. Alpert and Hopke (1980) reanalyzed the Boston data using target transformation 
factor analysis, and identified "soil" and "road dust" as major sources. Gaarenstroom et a1. (1977) analyzed Tucson aerosol data using factor analysis and found "soil" to be a significant source. Kowalczyk et al. (1982) used a CEB method on a set of aerosol data from the Washington, D.C. area and found contributions of $25 \%$ from "soil" and about $3 \%$ from "limestone".

In a series of studies for the Department of Energy and Natural Resources involving microscopic examination of hivol filters, the Illinois Institute of Technology Research Institute (IITRI) identified vehicle-raised "mineral particles" as the prime contributors to high TSP concentrations in Decatur and the Quad Cities (Arnold and Draftz, 1979), in Peoria (Arnold et al., 1980), and in East Moline and Milan, Illinois (IITRI, 1982). In a similar study, Lynn et al. (1976) found that about $65 \%$ of the mass of particles on hivol filters from 14 0.S. cities consisted of minerals such as quartz, calcite, hematite, and feldspars, and attributed them to such sources as wind erosion, resuspension of soil, quarrying, cement manufacturing, iron and steel production, and fuel combustion (flyash).

Evans and Cooper (1980) compiled an inventory of particle emissions from "open sources," including paved and unpaved roads, agricultural tilling and wind erosion, construction, surface mining, and tailings piles. Estimates of emissions were made for each state in the U.S. In 
Illinois, unpaved roads accounted for $57 \%$, wind erosion for $19 \%$, and tilling for $8 \%$ of such "open" emissions. In a subsequent paper, Evans and Cooper (1981) used this emission inventory to estimate the effects of these various open sources on TSP concentrations. In Illinois, unpaved roads accounted for $4.5 \mu \mathrm{g} / \mathrm{m}^{3}$, or about $6 \%$ of the statewide mean TSP concentration of $76.5 \mu \mathrm{g} / \mathrm{m}^{3}$. Other open sources could not be detected as contributing to measured TSP concentrations, due in part to the tendency for measurements to be made in urban, rather than rural areas.

The source apportionment techniques employed in the studies cited above all require multielement aerosol data as input. Other studies reporting such data for major U.S. cities include Leaderer (1978), for New York City, and Countess et al, (1980) for Denver. In addition, Smith et al, (1981) have reviewed concentrations, chemical composition, size distribution, and health effects of inorganic inhalable particles in Illinois.

The "crustal" dust identified in these various studies can come from wind erosion or tilling of agricultural soils, or from such man-made features as paved or unpaved roads, parking lots, construction, demolition, and surface mining. Thus, we also review here some basic information on soil erosion, and on vehicle-raised street dust. 


\subsection{WIND EROSION}

\subsubsection{Introduction and History}

Wind erosion is caused by a stong turbulent wind blowing across an unprotected soil surface. Although generally believed to be of consequence only in arid and semiarid areas, soil erosion by wind may occur wherever soil, vegetative, and climate conditions are conducive (U.N.F.A.O., 1960). These conditions exist when 1) the soil is loose, dry, and reasonably firely divided, 2) the soil surface is somewhat smooth and vegetative cover is absent or sparse, 3 ) the field is sufficiently large, and 4) the wind is strong enough to start soil movement.

Some soil from eroding fields becomes suspended and becomes part of the atmospheric dust load. Hagen and Woodruff (1973) estimated that eroding lands of the Great Plains contributed 244 and 77 million tons of dust to the atmosphere in the $1950 \mathrm{~s}$ and $1960 \mathrm{~s}$, respectively. Soil dust is an important class of aerosol that has the potential for long-range transport (Gillette, 1974; Clayton et al, 1972; Carlson and Prospero, 1972; Rahn et a1, 1979). Hidy and Brock (1970) estimated that dust raised by wind contributes $9.3 \%$ of the total production of aerosols 
based on an estimate of Judson (1968), or $21 \%$ of the total production if source estimates are adjusted to known aerosol composition (Gillette, 1974). Windblown soil dust obscures visibility (Patterson et al, 1976), pollutes the air, causes traffic hazards, fouls machinery, and impairs human and animal health.

3.2.2 Wind Erosion Processes

\subsubsection{Natural Wind}

Movement of soil particles is caused by wind forces exerted against the surface of the ground. The molecules of air actually in contact with a surface must be at rest, and (ideally) the airspeed increases logarithmically to a constant value at some distance from the surface. This region is called the "boundary layer." The variation of wind speed with height, within the boundary layer determines the magnitude of the shear stress or drag force exerted on the ground surface. The surface shear associated with the decrease in wind speed near the surface is a vertical transfer of horizontal momentum. Momentum decreases as the surface is approached. 
The minimum wind speed required to start soil movement is called the threshold speed. This speed depends on the size and weight of the soil particles and the friction provided by neighboring particles. In general, there are three types of pressures exerted on soil grains at the tbreshold of their movement by wind (Chepil and Woodruff, 1963; Chepil, 1959). Impact or velocity pressure is a positive pressure resulting from the impact of the fluid against the grain. This pressure causes the initial movement of the grain and varies directly as the square of the fluid velocity. The second type is a negative pressure on the lee side of the grain, known as viscosity pressure. Its magnitude depends on the fluid's coefficient of viscosity, density, and velocity. The third type of pressure is a negative pressure on the top of the grain, caused by the Bernoulli effect. Bernoulli's law states that wherever the fluid velocity is increased, as at the top of the soil grain, the pressure is reduced. This is called the static, isotropic, or internal pressure. This Bernoulli effect causes lift on the grain (Chepil, 1959). The impact or velocity pressure on a soil grain lying on the ground is known as form drag, and the pressure due to viscous shear in the fluid close to the surface of the soil grain is called skin friction drag. The sum of these two forces is the total drag (Chepil and Woodruff, 1963).

Chepil (1959) analyzed the drag, lift, and gravity forces on soil grains at the threshold of their movement by wind. Equilibrium between these forces and the soil grains was influenced by the diameter, shape, 
and density of the grains; the angle of repose of the grains with respect to the mi $n$ drag level of the fluid; the closeness of packing of the top grains; and the impulses of fluid turbulence associated with drag and lift (Bagnold, 1965; Cepil and Woodruff, 1963; Chepil, 1959; Lyles and Krauss, 1971). Experimental results (Malina, 1941; Chepil, $1945 \mathrm{a}, \mathrm{b})$ indicated that the minimum wind speed required to initiate movement of the most erodible particles -- those about $0.1 \mathrm{~mm}$ in diameter -- is about $16 \mathrm{~km} / \mathrm{hr}$ at a height of $30.5 \mathrm{~cm}$. The practical limit under field conditions where a mixture of sizes of single-grained materials is present is about $21 \mathrm{~km} / \mathrm{hr}$ at the same height. After soil particles start to move, they are carried by the wind in three types of movement -- saltation, surface creep, and suspension, depending upon their size in relation to the velocity and turbulence of the wind.

\subsubsection{Saltation}

This is the major mode of soil movement. Roughly 50-75\% of soil movement takes place via saltation (Chepil, 1945c). Particles in the size range from 0.1 to $0.5 \mathrm{~mm}$ diameter move in a bouncing or jumping action called saltation. After the initial motion, soil grains begin to roll along the surface under the direct impact pressure of the wind. It was observed that soil grains jumped vertically off a smooth surface after rolling downwind for a short distance (about $2-30 \mathrm{~cm}$ ) (Free, 1911; Bagnold, 1965; Chepil, 1945c). Bagnold (1965) and Chepil (1945c) 
explained that the causes of the vertical rise of particles in saltation is due to the spinning of the grains and the steep wind speed gradient near the surface. The air speed at any point near the grain is made up of two components, one due to the wind and the other due to the spinning of the grain. On the upper side of the grain, these two components act in the same direction, whereas below the grain they act in opposite directions. Thus, the speed is greater at the top surface than at the bottom. According to the Bernoulli effect, the pressure is decreased above and increased below the grain, so it is propelled upward. The Bernoulli effect, as mentioned earlier, is further intensified via the steep wind speed gradient that exists near the surface. Others (Lyles and Krauss, 1971; Bisal and Nielsen, 1962) observed that as the fluid threshold was approached, some particles began to vibrate, or rock back and forth. Erosive particles vibrated with increasing intensity as wind speed increased and then left the surface instantaneously as if ejected. Evidence supported the hypothesis that the particle-vibration frequency is related to the frequency band containing the maximum energy of the turbulent motion. The saltating particles rise almost vertically, rotating from 200 to 1000 revolutions per second, travel 10 to 15 times their height of rise, and return to the surface at forward and downind angles of 6 to 12 degrees (Woodruff et al, 1977; Chepil and Woodruff, 1963). When they strike the soil surface, they either rebound and continue their movement in saltation, or impart most of their energy by striking other grains, causing these grains to rise upward or roll along the surface. 


\subsubsection{Surface Creep}

The rolling or sliding of larger particles along the surface is called creep. These particles are too heavy to be lifted by the wind and are moved primarily by the impact of the particles in saltation, rather than by the direct impact force of the wind (Chepil, 1945c). They range in diameter from about 0.5 to $1.0 \mathrm{~mm}$, according to their density and the wind speed. Winds of extremely high speed may move particles larger than $1.0 \mathrm{~mm}$, but particles about 0.1 m diameter are the most erodible. Bagnold (1965) observed that at low wind speeds the grains move in jerks, a few at a time, but as the wind speed is increased the distance moved increases and more grains are set in motion, until in high winds the whole surface appears to be creeping forward. About 7 to $25 \%$ of soil movement occurs in this mode (Chepil, 1945c).

\subsubsection{Suspension}

This is the third type of soil movement, and may be characterized as the floating of small particles in the airsteam. Movement of these fine particles, smaller than $0.1 \mathrm{~mm}$, is usually initiated also by impact of particles in saltation. Fine dust particles generally must be kicked up 
by larger saltating grains, since they either do not protude into the turbulent air ayer or else they exhibit enough mutual cohesion to prevent being picked up directly by the wind. Once they enter the turbulent air layers, they can be lifted high into the atmosphere by rising eddies, and they are of ten carried a great distance before coming back to the earth's surface. Although only 3 to $38 \%$ of the soil movement is via suspension (Chepil, 1945c), this movement of fine particles is probably the most easily recognized characteristic of wind erosion.

\subsubsection{Effects on the Soil}

Once soil grains are loosened and soil movement begins, the impact of the jumping particles in saltation abrades the surface. This abrasion breaks down clods, destroys stable crusts, and wears down vegetative residues and living vegetation. Increasing numbers of particles are set in motion as erosion moves downind. Such as increasing soil flow is called soil avalanching (Chepil and Milne, 1941; Chepil, 1946a). The rate of soil flow is zero at the windward edge of an eroding field and increases with distance downwind until it reaches the maximum that a given wind can carry. The distance downwind at which the maximum rate of flow occurs varies with soil erodibility. The more erodible the soil, the greater the rate of avalanching, and the shorter the distance to maximum flow. 
The most serious consequence of wind erosion is the sorting action it causes. An eroding wind removes the fine porous particl- and leaves the coarser and denser particles behind (Moss, 1935; Daniel,1936; Chepil, 1957; Chepil, 1946b). Chepil (1957a) observed that the most distinct feature in the sorting process is the peak (predominant) diameter of the saltating grains. Fractions larger than the peak diameter tend to remain in the wind-eroded field; particles smaller than that diameter tend to be carried in suspension far through the atmosphere. The drifted materials derived from sand and loamy sand fields were composed of principally discrete, nonporous grains having an average bulk density of $2.37 \mathrm{~g} / \mathrm{cm}^{3}$. Their peak diameter was about 0.4 mm. The materials drifted from finer textured soils were predominantly aggregates with a distinct degree of porosity and an average bulk density of $1.70 \mathrm{~g} / \mathrm{cm}^{3}$. The peak diameter of this drifted material was about $0.6 \mathrm{~m}$ (Chepil, 1957a). Wind erosion sometimes virtually removes the surface soil (Chepil, 1946b, 1957a; Zingg, 1954; Chepil, 1957b). Very little sorting occurs on some fine-textured soils derived from loess. This non-selective removal by wind is associated primarily with loess sorted and deposited from the atmosphere during past geologic eras.

\subsubsection{Factors Affecting Wind Erosion}


Major factors that affect the amount of erosion from a given field are soil cloddiness, surface rouk less, wind speed, soil moisture, unsheltered field length, and vegetative cover.

\subsubsection{Soil Cloddiness}

Soil clods diminish wind erosion because they are large enough to resist the forces of the wind and because they shelter other erosive materials. Clods form during tillage. Their firmess and stability depend on soil mosture, compaction, organic matter, clay content, lime, and microbial activity. Clods are broken down by weathering, tillage, implement and animal traffic, and by abrasion (Woodruff et al, 1977). The size and bulk density of clods, and the proportion of clods to total soil material affect the erodibility (ease of detachment and transport) of a soil. From wind tunnel tests, Chepil (1956) determined relative erodibilities of soils relatively free from organic residues as a function of apparent specific gravity and of proportions of dry soil aggregates in various sizes. Clods larger than $0.84 \mathrm{~mm}$ in diameter were nonerodible. Since then, the nonerodible soil fraction greater than $0.84 \mathrm{~mm}$, as determined by dry sieving, has been used as a simple index of the wind erodibility of soils (Chepil and Woodruff, 1963; Chepil, 1957b). 


\subsubsection{Surface Roughness}

In addition to clods and soil aggregates, ridges and depressions formed by tillage operations also alter wind speed by absorbing and deflecting part of the wind energy away from the erodible soil. Chepil and Milne (1941), investigating the influence of surface roughness on intensity of drifting dune materials and cultivated soils, found that the initial intensity of drifting was always much less over a ridged surface than a smooth surface. Rough surfaces also trap saltating particles. This reduces abrasion and the normal buildup of eroding materials downwind. While the general effect of surface roughness is to reduce wind erosion, it also increases wind turbulence and exposes small areas to greater wind forces. Thus, excessive roughness may substantially reduce the benefits (U.N.F.A.O., 1960; Woodruff et al, 1977).

\subsubsection{Wind Speed and Soil Moisture}

Wind speed and soil moisture both affect wind erosion. The rate of soil movement by wind varies directly as the cube of wind speed (Bagnold, 1965; Chepil and Woodruff, 1963; Chepil, 1945c) and inversely as the cube of average soil moisture (Chepil, 1956). For convenience, 
wind speed and soil moisture are considered together as a local wind erosion climate factor (Chepil et al, 1962). It is an incex of average rate of soil movement by wind as influenced by moisture content in surface soil particles and average wind speed.

\subsubsection{Unsheltered Field Length}

As mentioned earlier, rate of soil flow increases with distance downwind across an eroding field. Erosive winds vary highly in direction and seldom follow field boundaries. Therefore, the amount of soil lost from a given field cannot be determined from the width or length of the field alone. Thus, field length was initially considered as the distance across a field in the prevailing wind-erosion direction. Also, if any barrier is present on the windward side of the field, the distance it fully shelters the land from the wind must be subtracted from the total distance across the field along the prevailing direction. However, sometimes there is essentially no prevailing wind-erosion direction. Therefore, the preponderance of wind-erosion forces in the prevailing wind-erosion direction is now used to assess equivalent field length (Skidmore and Woodruff, 1968; Skidmore, 1965). 
3.2.3.5 Vegetative Cover

Good vegetative cover on the land is the most permanent and effective way to control wind erosion (Woodruff et a1, 1977). Living or dead vegetative matter protects the soil surface from wind action by reducing wind speed and preventing much of the direct wind force from reaching erodible soil particles. It also reduces rates of erosion by trapping soil particles, which prevents the normal avalanching of soil material downwind. Protection depends on the amounts (Chepil, 1944; Chepil et al, 1963; Siddoway et al, 1965), the size (Siddoway et al, 1965; Woodruff and Siddoway, 1965), and the orientation (Chepil et al, 1955; Englehorn et al, 1952; Skidmore et al, 1966) of the residue. In general, the finer the residue or the higher the residue stands above the ground, the more it slows the wind and the more it reduces wind erosion. Standing stubble with rows perpendicular to the wind controlled wind erosion much more effectively than did rows parallel to the wind direction (Englehorn et al, 1952). Recent research (Lyles et al, 1974) indicates that if residue is standing and is equidistantly spaced, much less residue is needed to control wind erosion than has been reported previousiy. 


\subsubsection{Wind Erosion Equation}

Studies to understand the wind erosion process, identify major factors influencing wind erosion, and develop wind erosion control methods led to the development of a wind erosion equation (Niles, 1961; Chepil and Woodruff, 1963; Woodruff and Siddoway, 1965). The relationship between average soil loss in tons per acre by wind erosion from a given field (E) and the five factors influencing wind erosion can be expressed as $E=f\left(I^{\prime}, K^{\prime}, C^{\prime}, L^{\prime}, \nabla\right)$, where $I^{\prime}$ is the soil erodibility index, $\mathbb{K}^{-}$is the soil surface roughness factor, $C^{\prime}$ is the climatic factor, $\mathrm{L}^{\prime}$ is the unsheltered field length along the prevailing wind direction, and $\nabla$ is the equivalent quantity of vegetative cover.

The soil erodibility index is the percentage of soil aggregates larger than $0.84 \mathrm{~mm}$. This information can best be obtained by dry sieving. However, in practice the value is often estimated from wind-erodibility groups (Hayes, 1972), which are based on the soil type or the predominant soil texture class. The surface roughness factor can be determined from the chart given by Skidmore and Woodruff (1968). The climate factor can be obtained from maps given by the same reference, which indicate an approximate value for any location in the U.S. and the agricultural areas of Canada. The unsheltered field length factor can be determined from 1 ) the angle of deviation of the prevailing 
wind-erosion direction from right angles to a field strip, 2) the preponderance of wind erosion forces in the prevailing wind-erosion direction, 3) the height of the wind barrier, and the field length. Prevailing wind erosion directions, magnitudes, and the preponderence of wind erosion forces are available for 212 locations in the United States (Skidmore and Woodruff, 1968). Other factors can be measured in the field. The equivalent quantity of vegetative cover expressed the effects of quantity, kind, and orientation of vegetative cover on wind erosion. Values of $\nabla$ are available for most crops (Skidmore and Woodruff, 1968; Siddoway et al, 1965; Woodruff and Siddoway, 1965; Craig and Tupelle, 1964). This equation bas been used extensively by the Soil Conservation Service, USDA, in designing control practices and determining crop tolerance to wind erosion (Hayes, 1965, 1966). Other applications include: 1) predicting horizonal soil fluxes to compare with vertical aerosol fluxes (Gillette et al, 1972), 2) estimating fugitive dust emissions from agricultural and subdivision land (Wilson, 1975), and 3) estimating effects of wind erosion on productivity (Lyles, 1975).

Charts, tables, and other supplementary information needed to solve the wind erosion equation were given by Skidmore and Woodruff (1968). Examples of field applications of the equation were given by the same authors and by Moldenhauer and Duncan (1969). A computer program for solving the equation is also available (Skidmore et al, 1970; Fisher and Skidmore, 1970). 


\subsubsection{Summary}

This section summarizes basic information available on wind erosion. Wind erosion is caused by a strong turbulent wind blowing across an unprotected soil surface that is smooth, bare, loose, dry, and finely divided. Wind erosion not only sorts soil material, removing silt, clay, and organic matter, but it also sandblasts and destroys crops. The suspended dust also obscures visibility and pollutes the air.

Soil particles start to move when wind forces overcome gravity. They are carried by wind in three modes of movement -- saltation, surface creep, and suspension. The saltating grains move in a bouncing, spinning, and jumping manner. When they strike the soil surface, they break down clods and crusts, and dislodge other erodible particles. Movement in suspension and in surface creep is a result of movement in saltation. Therefore, control of wind erosion depends on reduction or elimination of saltation. Major factors affecting wind erosion are soil cloddiness, surface roughness, wind speed, soil moisture, field length, and vegetative cover. Studies of the influence of these factors on wind erosion led to development of the wind erosion equation. 
The general principles of wind erosion control include production of a greater fraction of clods larger than $0.84 \mathrm{~mm}$, roughening the land surface, establishing wind barriers or soil traps at intervals, reducing field length, and providing vegetative cover or residues.

\subsection{EMISSIONS FROM PAVED AND UNPAVED ROADS}

In contrast to wind erosion of soils, for which physical mechanisms have been discovered, resuspension of dust from paved and unpaved roads has been investigated only to the point of obtaining empirical methods of estimating emissions. Evans and Cooper (1980) summarized the empirical formulas they used to estimate particle emissions from a wide variety of open sources. The results of Cowherd et al, (1974) were used to estimate emissions from unpaved roads.

Cowherd and his colleagues at the Midwest Research Institute have also provided formulas for estimating emissions from paved roads (Cowherd and Mann, 1976; Cowherd and Englehart, 1982).

Through analysis of TSP data from 20 sites over a period of more than 8 years, Newman et al, (1976) estimated the relative contributions of wind and traffic to resuspension of urban street dust in Chicago. 
3.4 COMPARISON OF AEROSOL MEASUREMENT METHODS

As new methods of sampling are adopted, it is important to compare the new and old sampling methods in side-by-side operation. Kolak and $\nabla$ isalli (1981) reviewed the recent literature comparing various size-selective samplers with the currently standard high-volume sampler. Samplers that exclude particles larger than about 15-20 $\mu$ m collect only about 0.5 to 0.8 as much mass as the standard hivol sampler. Kolak and Visalli obtained very similar results in the Buffalo, NY area. Countess et al, (1980) compared the total mass collected by a dichotomous sampler with a $15 \mu$ m cutoff with that collected by a standard hivol. They found a mean ratio of 0.44 with a standard deviation of 0.18 over a 40 -day period in November and December, 1978, at Denver.

\section{EXPERIMENTAL METHODS}

\subsection{SAMPLER LOCATIONS}

The rural samplers were located at the Water Survey's Bondville Road field site, about $10 \mathrm{~km}$ southwest of Champaign. The site is a 3.0 hectare ( 7.5 acre) grassy field, surrounded by fields of corn and 
soybeans. Precipitation and aerosol sampling are also carried out at this site in support of our study of air pollution scavenging for the U.S. Department of Energy (DOE). This site is also a precipitation sampling site for both the DOE/EPA Multistate Atmospheric Power Production Pollutants Study (MAP3S) network and the National Atmospheric Deposition Program (NADP).

A map of a portion of Champaign, showing the aerosol sampling sites within the NURP study basins, is shown in Figure 1. Three hivol samplers were located in the commercial "Mattis South" basin along Mattis Avenue. As shown in the figure, two samplers were located on the west side of Mattis Avenue, and one on the east side. The dichotomous sampler was located adjacent to the hivol on the east side of Mattis Avenue. An additional hivol sampler was located in a residential Champaign neighborhood; this is designated the John Street site in Figure 1.

The hivol samplers on the east and west sides of Mattis Avenue were all located $7.0 \mathrm{~m}( \pm 0.4 \mathrm{~m})$ from the curb to minimize any effects of distance from the street on the concentrations measured by the various samplers. At the John Street site the hivol was $17.6 \mathrm{~m}$ north of the curb. 


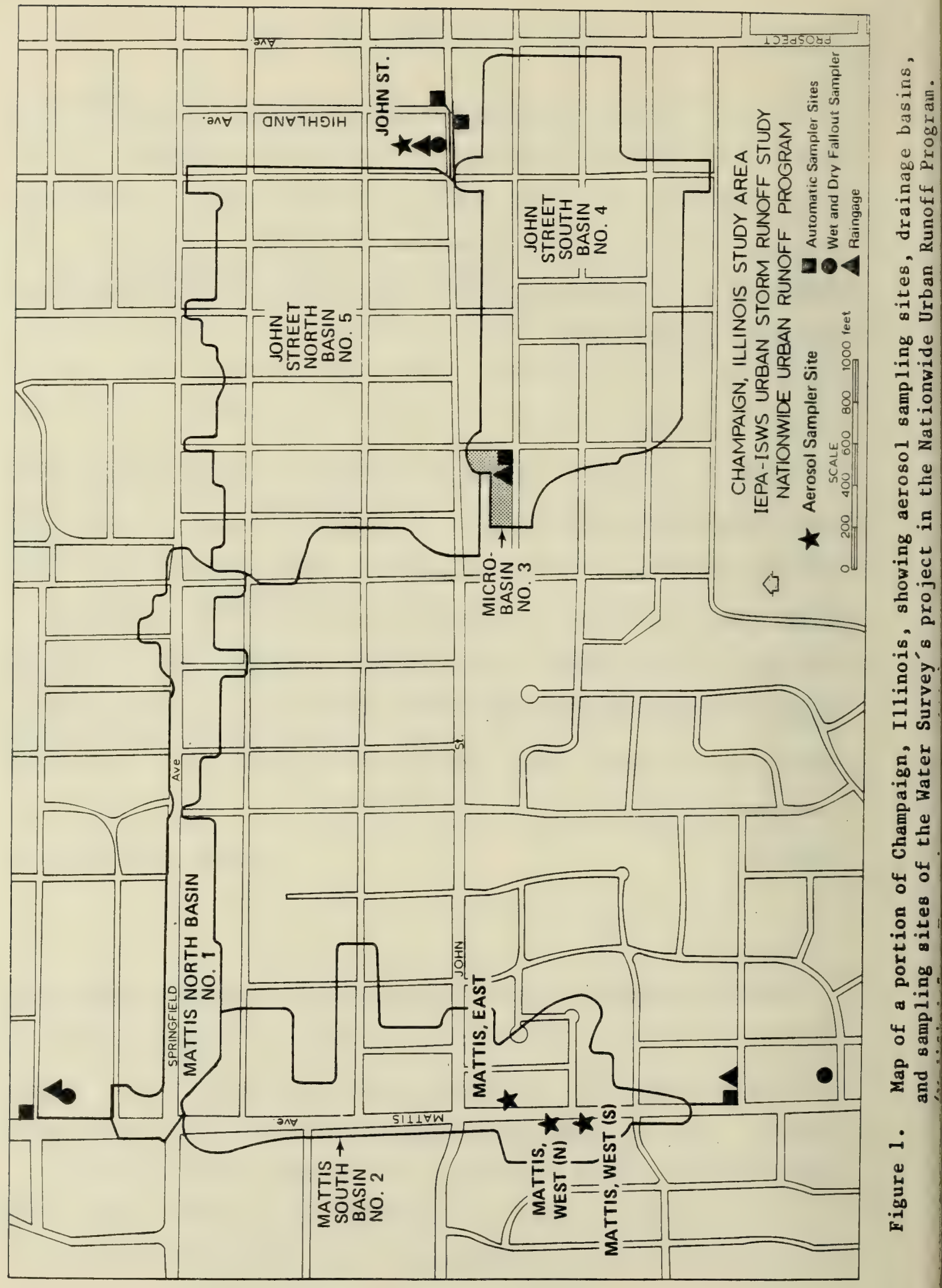




\subsection{SAMPLING METHODS}

A11 the aerosol samplers were installed so that their inlets were 2 m above ground level. Thus, they conformed to the standard height, i.e., between 2 and $15 \mathrm{~m}$, at which hivol inlets must be placed. The urban hivol samplers were all positioned so that their inlets faced the street. It was important to be consistent on this detail since there is evidence (Wedding et al., 1977; Ortiz, 1978) that hivol sampling effectiveness varies with sampler orientation to the air flow.

The IEPA provided the standard hivol samplers and calibrated them prior to our use. Further, IEPA provided replacement pumps when needed. The IEPA also provided glass fiber filters for the hivol samplers, weighed the filters before and after exposure, and calculated TSP concentrations.

The urban hivol filters were changed at approximately 9:00 a.m. each Monday through Friday. This schedule provided five 24-hr duration filters each week from each sampler. The first filter of the week began Monday morning and the last ended Saturday morning. At the rural site, hivol filters were changed on Monday, Wednesday, and Friday mornings (about 10 a.m.); here also, the filters were exposed for $24 \mathrm{hr}$. 
The dichotomous sampler collected a pair (fine and coarse) of 24-hr duration filters each day of the week. The "fine" filter collected particles less than approximately $2.5 \mu \mathrm{m}$, and the "coarse" filter those between 2.5 and about $15 \mu \mathrm{m}$ (aerodynamic diameter). The upper size limit on the coarse particles is known to vary between 12 and $18 \mu \mathrm{m}$ depending on wind speed, for speeds less than $20 \mathrm{~km} / \mathrm{hr}$ (Spengler et al, 1981). Each sample began and ended at 9:00 a.m. As suggested in the operations manual for the sampler (Spengler et al. , 1981), the instrument was carefully checked for leaks and calibrated prior to use. Sampling in Champaign began in June, 1981, and ended in October, 1981. During normal operations, the dichotomous sampler was recalibrated every 2-4 weeks to insure accurate sample volume determinations.

\subsection{ANALYSIS METHODS}

As mentioned above, TSP measurements were made by the IEPA, using standard methods.

The Teflon (TM) filters used in the dichotomous sampler were weighed before and after use to measure total aerosol mass. Unfortunately, sporadic difficulties with the balance used for these measurements caused the filter weights to be unreliable for the samples collected at the rural site. Thus, total aerosol mass concentrations for the 
dichotomous filters are available only for the samples collected in Champaign.

Multielement analyses were carried out on the fine and coarse dichotomous filters by NEA, Inc., Beaverton, Oregon, using X-ray fluorescence methods. The analysis technique was capable of detecting Al, Si, P, S, Cl, K, Ca, Ti, $7, \mathrm{Cr}, \mathrm{Mn}, \mathrm{Fe}, \mathrm{Ni}, \mathrm{Cu}, \mathrm{Zn}, \mathrm{As}, \mathrm{Se}, \mathrm{Br}, \mathrm{Rb}$, $\mathrm{Sr}, \mathrm{Cd}, \mathrm{In}, \mathrm{Sn}, \mathrm{Sb}$, and $\mathrm{Pb}$. Laboratory blanks were measured for the purposes of correcting the measurements for filter impurities. Field blanks were analyzed with each batch of samples to insure that the filters were not contaminated during handling or while stored in the sampler before or after exposure. Standards were analyzed with every batch to insure accuracy.

\subsection{STREET SWEEPING OPERATIONS}

The street sweeper made single passes along both east and west curbs of the Mattis South basin once per week, normally on Tuesday morning, between 15 April and 26 May 1981. The sweeper used was a 1973 Elgin, Model Pelican "S," a three-wheeled mechanical sweeper with right and left side gutter brooms and a main rotary broom. Its sweeping path using one outside broom was $2.4 \mathrm{~m}(8 \mathrm{ft})$ wide. 
Measurements of collection efficiency by Bender (1982) indicated that the sweeper collected about $67 \%$ of the total dust mass on the street. This approximate efficiency was maintained for all particle sizes down to $125 \mu \mathrm{m}$, below which efficiency decreased markedly. Other measurements by Bender (1982) indicated that $46 \%$ of the total dust mass was in particles greater than $1000 \mu$ m in diameter.

In six sweepings of the Mattis South basin, the median mass collected was $28.8 \mathrm{~g} /$ curb $\mathrm{m}(102 \mathrm{lb} / \mathrm{curb} \mathrm{mi})$. The maximum collection was $44.5 \mathrm{~g} /$ curb m $(158 \mathrm{lb} /$ curb $\mathrm{mi})$ and the minimum $13.2 \mathrm{~g} / \mathrm{curb} \mathrm{m}$ (47 $\mathrm{lb} /$ curb mi).

\subsection{SUPPORTING DATA}

Several kinds of data were also collected to aid in interpretation of the air quality data. These include meteorological data, specifically winds and precipitation, as well as information on traffic densities and the dates when the streets were swept.

Precipitation was measured at the Mattis Avenue and John Street raingage sites, as shown in Figure 1, and provided to us, along with traffic counts and dates of street sweeping, by the Water Survey NURP 
project mentioned earlier. Winds applicable to the rural site were measured at The University of Illinois Willard Airport, $8 \mathrm{~km}$ east of the rural site. Urban wind measurements were made at the Water Survey Headquarters, about $4 \mathrm{~km}$ east of the Mattis Avenue sites.

\subsection{SOURCE APPORTIONMENT BY CHEMICAL ELEMENT BALANCE}

The method used was described by Dzubay et al, (1980), which uses the weighting factor suggested by Watson (1979). Watson's weighting factor has the advantage that it includes uncertainties in both ambient elemental concentrations and the elemental composition of the sources.

The method assumes that the aerosol composition can be described by:

$$
P_{k}=\sum_{j} C_{j k} M_{j}
$$

where

$P_{k}$ is the predicted concentration of element $\mathrm{k}\left(\mathrm{ng} / \mathrm{m}^{3}\right)$,

$c_{j k}$ is the fractional abundance of element $k$ in source $j$,

$M_{j}$ is the total mass concentration of source $j\left(\mathrm{ng} / \mathrm{m}^{3}\right)$, and the sum is over all sources $j$. 
The elemental abundances of any component $j$ must be normalized so that

$$
\sum_{\mathrm{k}} C_{j k}=1
$$

where the sum over $k$ includes all elements that contribute significant mass to the aerosol.

The source concentrations $\mathrm{M}_{j}$ are obtained by linear least squares, in which $x^{2}$ is minimized in the expression

$$
x^{2}=\Sigma\left(0_{k}-P_{k}\right)^{2} / \sigma_{k}^{2}
$$

where $O_{k}$ is the observed concentration of element $k$ and $\sigma_{k}$ is Watson's weighting factor, given by

$$
\sigma k^{2}=a_{k}{ }^{2}+\sum_{j} b_{j k}{ }^{2} M_{j}{ }^{2}
$$


where $a_{k}$ represents the uncertainties in ambient concentrations and $b_{j k}$ the uncertainties in the source compositions.

The solution to Equations (3) and (4) was reached after three iterations. The first iteration calculated the weights ${ }{ }_{k}$ using $M_{j}=0$. The next two iterations calculated the weights using the values of $\mathrm{M}_{j}$ from the previous iteration. The sum in Equation (3) included only those elements $k$ for which a fit was demanded. The elements used in the fit were varied in this work, and the variations are discussed later, with the results.

5 RESULTS AND DISCUSSION

5.1 RURAL MEASUREMENTS

5.1.1 Comparison of Inhalable and Total Aerosols

A comparison of particle mass concentrations measured by the standard and size-selective hivols is shown in Figure 2. The solid line represents perfect agreement between the two samplers. It is clear that the standard hivol commonly measures higher concentrations than the 


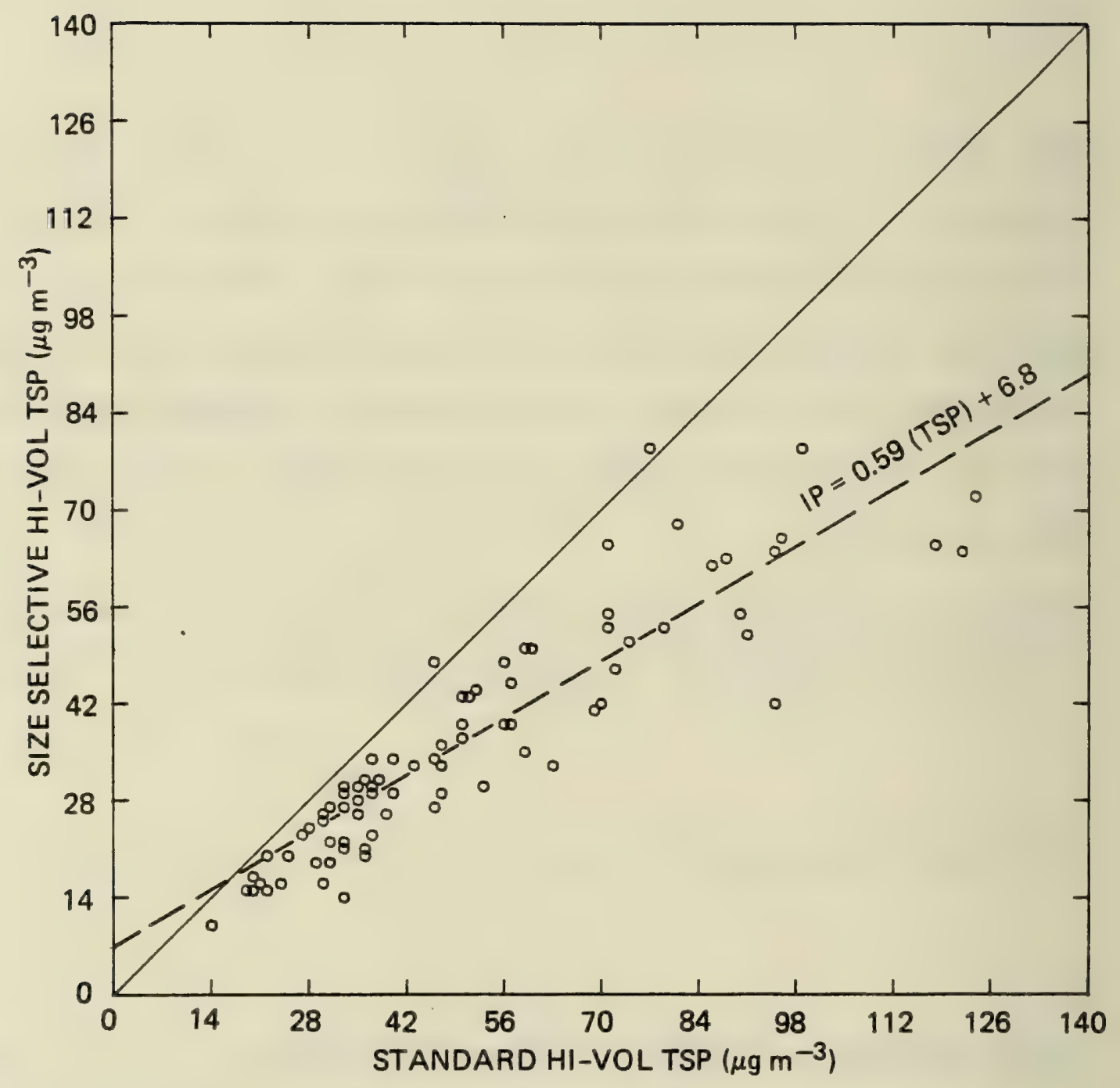

Figure 2. Comparison of rural particle mass concentrations measured by standard and size-selective high volume samplers. 
size-selective hivol, which excludes particles larger than $15 \mu \mathrm{m}$ aerodynamic diameter. The dashed line in Figure 2 is the least squares linear regression line fitted to the data: IP $=0.59$ (TSP) +6.8 . In this equation, IP is the inhalable particulate concentration, as measured by the size-selective hivol, and TSP is the total suspended particulate concentration, as measured by the standard hivol. Thus, when the standard hivol measures a TSP concentration of $100 \mu \mathrm{g} / \mathrm{m}^{3}$, the size-selective hivol would measure, typically, a IP concentration of 66 $\mu \mathrm{g} / \mathrm{m}^{3}$.

Table 1 lists comparisons between TSP and IP from the literature as well as from this study. In the cases of the four previously published results, IP was measured with dichotomous samplers. However, all the samplers used had upper cutoffs of approximately $15 \mu$ m aerodynamic diameter, the same as the size-selective hivol used in the rural phase of this study, so comparisons are valid. 
Table 1. Comparison of inhalable particle/total particle ratios in this study with literature values.

\begin{tabular}{ccc} 
Site No. of & -- & - \\
Reference & locations sites & $N^{*}$ \\
\hline
\end{tabular}

$\begin{array}{lcccccc}\text { Pace and Meyer, } 1979 & \text { urban } & 10 & * * & 0.5 & -0.7 & \\ \text { Spengler et al, } 1980 & \text { urban } & 4 & * * & & 0.59-0.66 \\ \text { Countess et al, } 1980 & \text { urban } & 1 & 16 & & 0.44 \\ \text { Kolak and Visalli, } 1981 & \text { urban } & 4 & 17 & 0.58-0.64 & \\ & \text { rural } & 1 & 21 & 0.80 & \\ \text { This study } & \text { rural } & 1 & 98 & 0.74 & 0.76 \\ & \text { urban } & 1 & 95 / & 0.50 & \end{array}$

* Approximate number of observations per site.

** Not known. 
The table lists two measures of the IP/TSP ratio. The first, $\overline{I P} / \overline{T S P}$, is the ratio of the mean values of IP and TSP, while the second, $\overline{\text { IP/TSP, }}$ is the mean of all the ratios computed separately for each pair of samples. (Mean values are indicated by bars over the quantity.)

The four studies of inhalable/total ratios in urban areas found ratios in the range 0.4 to 0.7 , whichever form of the ratio is used. Our urban value of $\overline{\mathrm{IP} / \overline{\mathrm{TSP}}}(0.50)$ falls within this range.

Kolak and Visalli (1981) compared results at one rural site southwest of Buffalo to four urban sites in the Buffalo area, and found higher IP/TSP values in the rural area. Since these authors measured IP with a dichotomous sampler, they were able to deduce that the urban-rural differences were probably caused by differences in the measured fine particle concentrations. These differences might represent real differences in airborne particle concentrations, but the authors note that they could have been caused by various sampling difficulties, such as artifact sulfate formation or a variation in sampling efficiency with particulate loading.

Our rural measurements, based on 98 pairs of samples, gave an IT/TSP ratio slightly smaller that Kolak and Visalli's rural value, but higher than any of the literature values for urban areas. Our rural $\overline{\text { IP/TSP }}$ 
ratio was also somewhat higher than the two published urban values. Thus our results agree quite well with previous measurements in a rural area near Buffalo, New York. Kolak and Visalli used "standard" measurement techniques, but did not state the height above ground for their sampler inlets. Since the standard height for sampler inlets is 2-15 $\mathrm{m}$, it is not possible to tell what height was used for the Buffalo area measurements, or how it compares to our inlet height of $2 \mathrm{~m}$.

\subsection{URBAN HIVOL MEASUREMENTS}

\subsubsection{Data Sunmary}

An illustration of a small portion of the available data, plotted against time, is given in Figure 3 . The data include TSP measurements at four sites, along with daily rainfall amount, dates of street sweeping, and winds. Wind directions are shown by direction category. The westerly wind category ( $W$ in Figure 3 ) includes days when the hourly mean wind direction was from the 203 to 338 degree sector for at least $75 \%$ of the hours between $6 \mathrm{a} \cdot \mathrm{m}$. and midnight. Wind direction was not considered relevant in the remaining hours of the sampling period because only a very small fraction of the traffic occurs then. Similarly, the easterly wind category (E) includes days when the hourly mean wind direction was from the 23 to 158 degree sector at least $75 \%$ of 

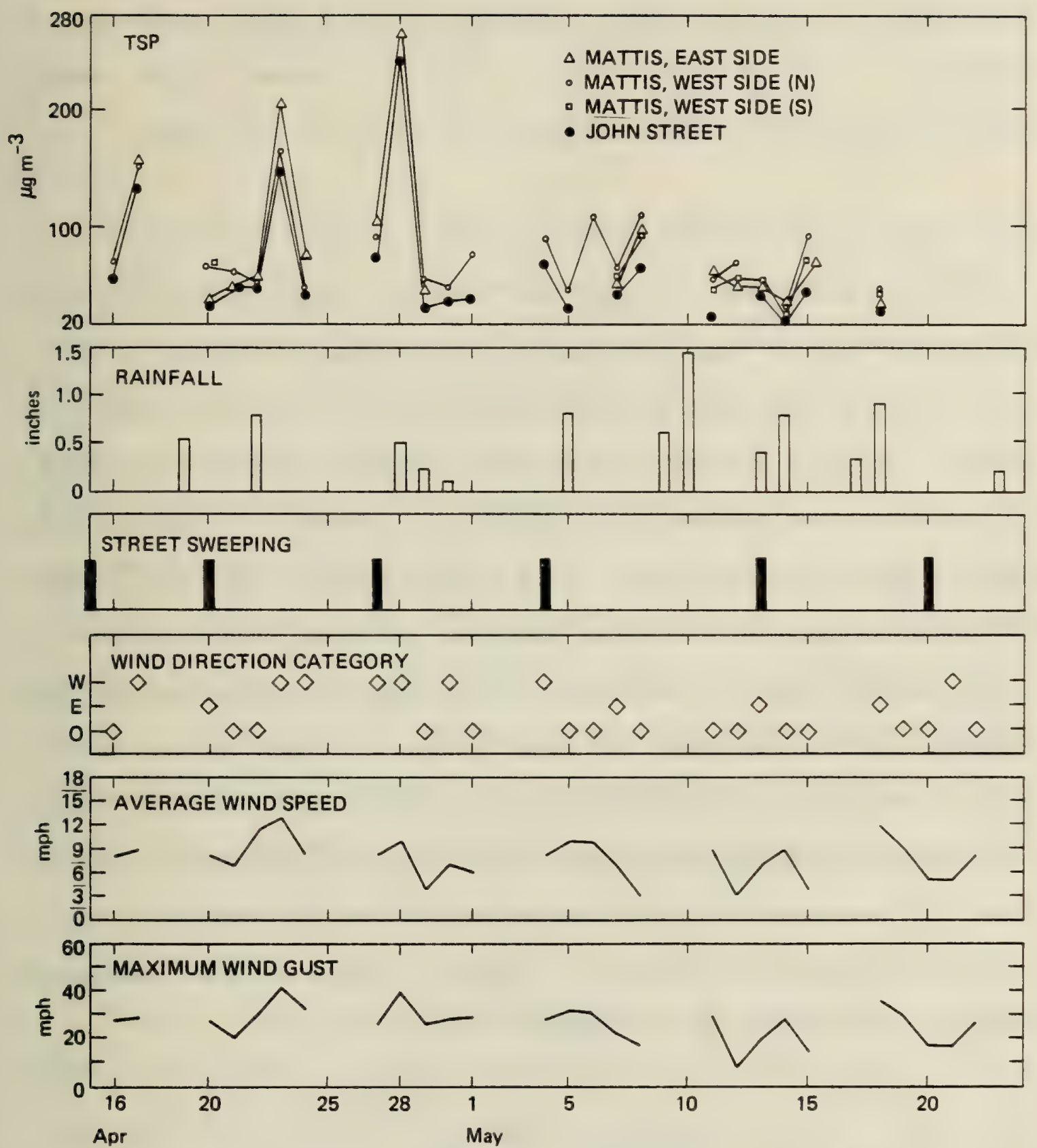

STARTING DATE FOR FILTERS

Figure 3. Illustration of data collected in 1981 for evaluation of effects of street sweeping on air quality. 
the hours in the same time interval. The "other" wind direction category ( 0 ) includes all other days. Mean wind speed during sample collection and daily maximum 1-minute gust speed are also shown.

Figure 3 shows that rainfall was frequent and relatively heavy during the period shown, with seven days having $1.27 \mathrm{~cm}(0.5 \mathrm{in.})$ or more. Streets were swept at approximately weekly intervals during the period. Figure 3 includes only a small fraction of the data collected, but it illustrates several relationships that occurred throughout the sampling period. One of these is the generally high correlation between TSP observations from the several samplers, including the one located in a residential area. Generally, on days when TSP concentrations were unusually high at one site, they were unusually high at all sites. There were still differences in concentration between sites, of course. A very obvious difference in Figure 3 was that the residential (John) site had consistently lower concentrations than the commercial sites. Differences between collectors on opposite sides of the commercial roadway are also apparent in Figure 3.

\subsubsection{Effects of Street Sweeping}


5.2.3 TSP Concentrations Upwind and Downwind of Mattis Avenue

In assessing possible effects of street sweeping on air quality, it was first necessary to establish whether the test roadway was indeed a source of aerosols. To examine this question, we divided the data according to the wind direction categories mentioned earlier. This was done to assess which samplers were upwind, and which downwind, when the wind direction was either easterly or westerly (i.e., having a large cross-wind component, relative to Mattis Avenue) and to identify those days when the wind (1) was from either north or south, or (2) blew from the east for a portion of the sampling period and from the west for another portion of it, so that it was difficult to assign upwind and downwind directions. Results for the east and west wind cases are shown in Figure 4, and those for the "other" winds in Figure 5.

Figure 4 includes only measurements made in easterly or westerly winds, and shows a clear tendency for the downwind samplers to have higher TSP values for both of these direction classes. The upwind samplers experienced higher TSP concentrations with westerly winds than with easterly winds. For example, the upwind means were $71 \mu \mathrm{g} / \mathrm{m}^{3}$ for 26 days with west winds and $60 \mu \mathrm{g} / \mathrm{m}^{3}$ for 30 days with east winds, a difference of $18 \%$, relative to the east wind value. For both east and west winds, however, the downwind samplers had higher TSP values, 


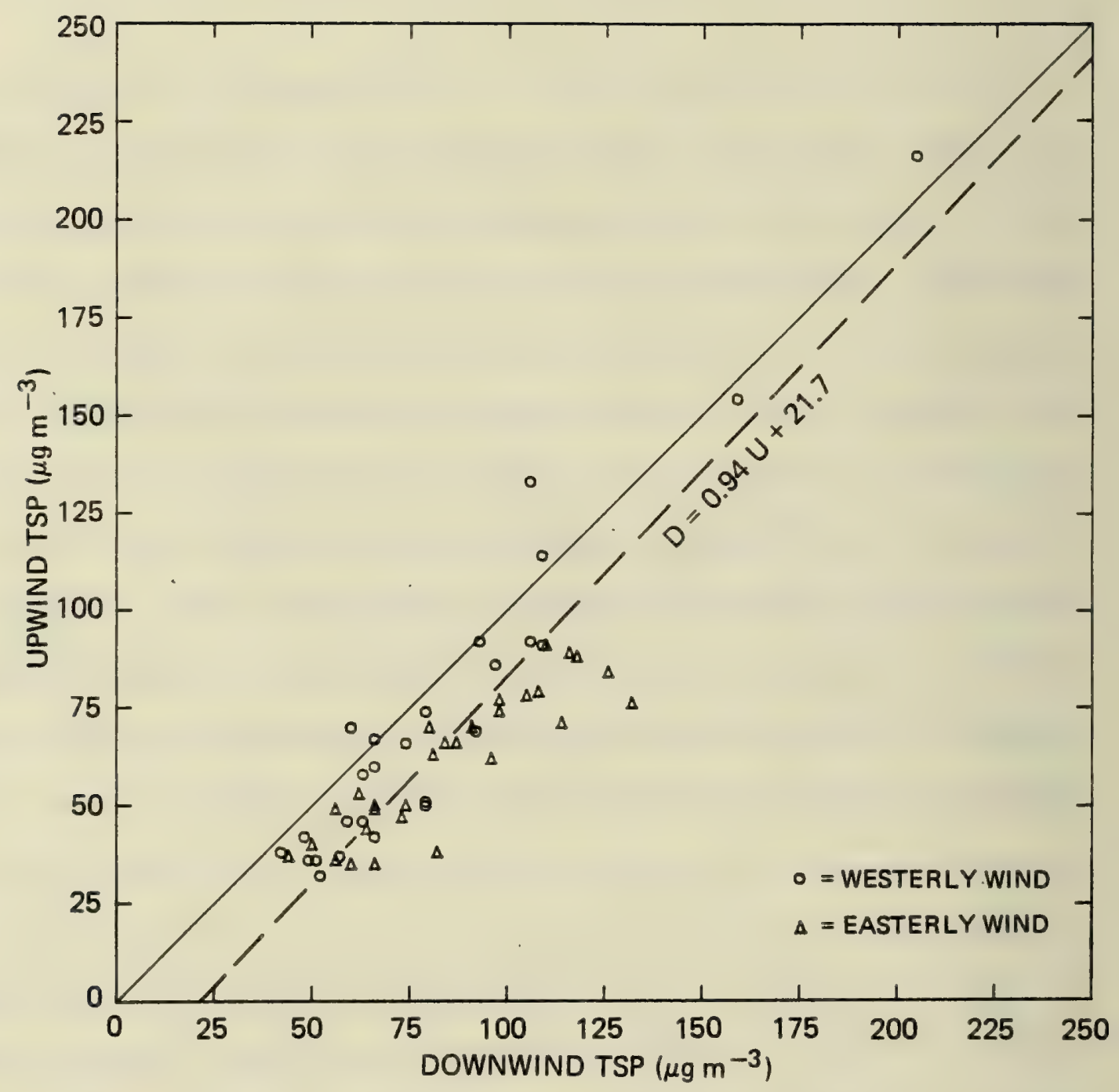

Figure 4. Comparison of TSP concentrations upwind and downwind of Mattis Avenue on days when winds were predominantly from the east or west. 


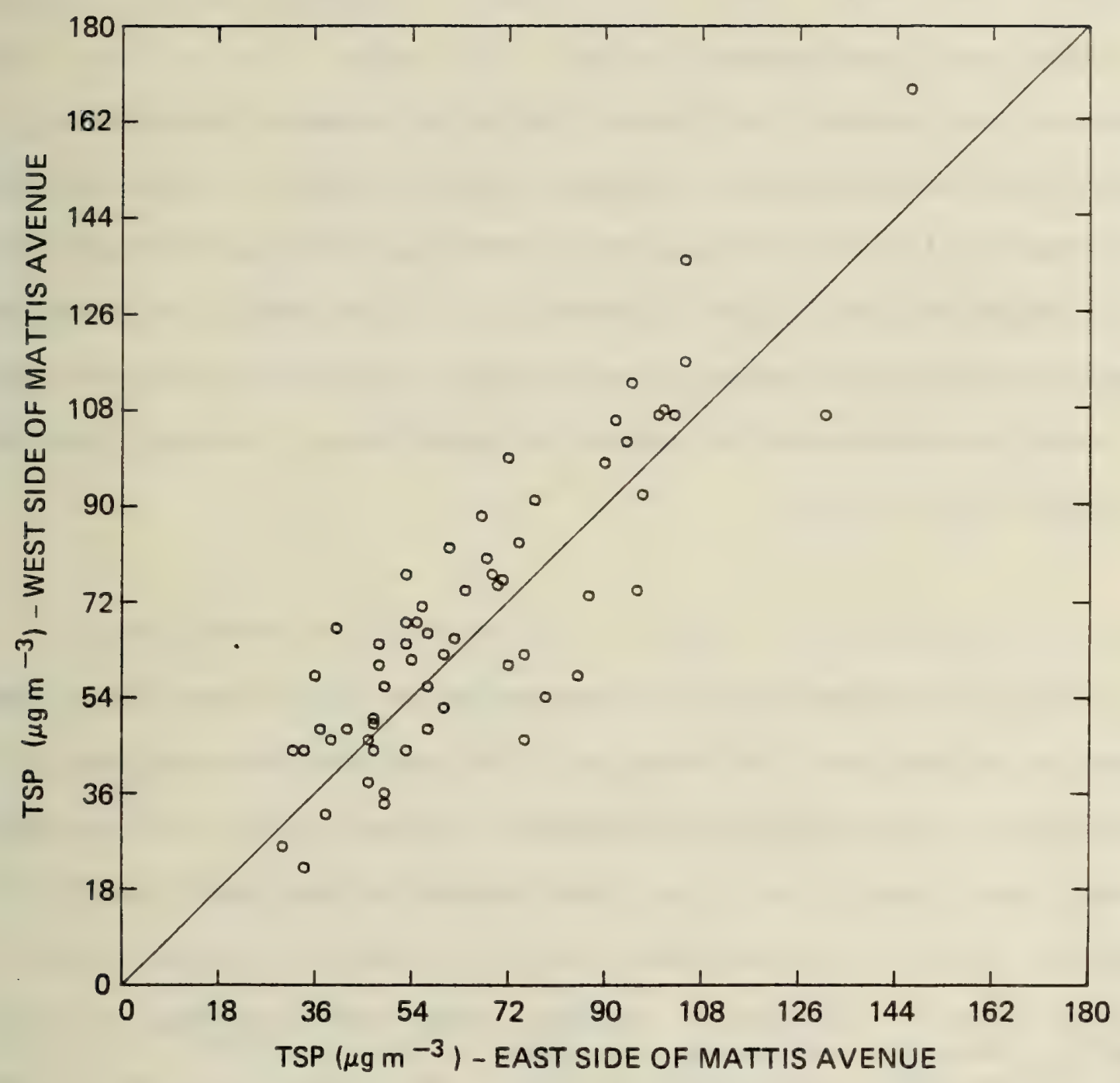

Figure 5. Comparison of TSP concentrations on the east and west sides of Mattis Avenue on days when winds were not predominantly from the east or west. 
clearly showing the road to be a source of airborne particles. As before, the solid line in the figure represents perfect agreement between upwind and downwind samplers. The dashed line is the least squares regression line for estimating the downwind concentration, D, from the upwind value, $U: D=0.94(U)+21.7$. Since the slope of the regression line is close to 1.0 , the intercept, $21.7 \mu \mathrm{g} / \mathrm{m}^{3}$ gives a good indication of the extra airborne dust concentration that may be attributed to the roadway. The slope near 1.0 shows that the added TSP concentration caused by the roadway is approximately constant for all upwind concentrations.

To consider the possibility that the differences in mean upwind TSP concentrations were the result of differences in mean wind speed, the following wind speed data are presented. The mean value of the daily mean wind speed, for 26 west wind samples, was $6.4 \mathrm{~m} / \mathrm{sec}(\mathrm{S} . \mathrm{D} .=2.0$ $\mathrm{m} / \mathrm{sec}$ ), while the corresponding value for 30 east wind samples was 5.0 $\mathrm{m} / \mathrm{sec}$ ( $\mathrm{S} . \mathrm{D} . \quad=2.1 \mathrm{~m} / \mathrm{sec}$ ). Analogous means for the maximum gusts were $24.0 \mathrm{~m} / \mathrm{sec}(\mathrm{S} . \mathrm{D} .=8.6 \mathrm{~m} / \mathrm{sec})$ for west wind samples and $18.2 \mathrm{~m} / \mathrm{sec}$ (S.D. $=5.4 \mathrm{~m} / \mathrm{sec}$ ) for east wind samples. Thus, the relative differences in mean wind speed (28\%) and mean maximum gust speed (32\%) between easterly and westerly winds were somewhat greater than the relative differences in TSP concentrations, but wind speed differences cannot be ruled out as a possible cause. Another possible cause is that the site was closer to agricultural sources with west winds, since it was located near the western edge of the Champaign-Urbana metropolitan 
area.

Beside differences in upwind TSP between east and west winds, close examination of Figure 4 also suggests systematic differences in the upwind/downwind differences between east and west winds. Although the overall upwind/downwind difference was $21.7 \mathrm{\mu g} / \mathrm{m}^{3}$, in 26 west wind cases the upwind and downwind means were 73 and $82 \mu \mathrm{g} / \mathrm{m}^{3}$, respectively, which is a difference of only $9 \mu \mathrm{g} / \mathrm{m}^{3}$, or $11 \%$ of the downwind mean.

Figure 5 shows the relationship between TSP concentrations on the east and west sides of Mattis Avenue for the "other" wind category. There was no strong tendency for either side of the street to have higher values, as would be expected in the absence of a strong wind component perpendicular to the street.

5.2.4 TSP Concentrations in Commercial and Residential Areas with and without Sweeping

The evidence presented thus far makes it clear that the street was indeed the source of airborne particles, since the downwind sampler had higher concentrations in both east and west winds, but neither side had predominantly higher concentrations with "other" winds. Next we address 
the question of whether street sweeping had any effect on reducing the strength of this source. This is done by comparing downwind-upwind differences during periods with and without regular weekly sweeping. A day was considered to be in the "swept" period if the street was swept on that day or any of the previous seven days, and in the "unswept" period otherwise. A second comparison examines commercial-residential differences with and without weekly sweeping (by the same criteria) at the commercial site. (The residential site was not in one of the experimental or control basins, and thus was swept irregularly, at intervals of 2-4 weeks, during the sampling period.)

Table 2 presents results of a two-way analysis of variance (Brown, 1977 ) on downwind-upwind differences ( $\triangle$ TSP1). It examines both sweeping and time as sources of variance. The upper part of the table gives mean downwind-upwind differences in TSP concentrations for swept and unswept periods in both "spring" (up to 30 June) and "summer" (after 30 June). There appear to be differences between seasons (with higher concentrations in summer than in spring), but not between periods of sweeping and not sweeping. The analysis of variance table in the lower part of the table confirms that there were no significant differences between periods of sweeping and no sweeping, but also indicates that the differences between seasons were significant at the 0.10 level, but not at the 0.05 level. Table 3 presents results of a similar analysis of variance on commercial-residential TSP differences ( $T$ TSP2). Again, differences between seasons appear large (with concentration differences 
Table 2. Results of 2-way analysis of variance on upwind-downwind TSP differences $\left(\mu g / \mathrm{m}^{3}\right)$ across the test roadway.

Swept Unswept

Number of cases

Mean

S.E.M.

Source of variance

Time

Sweeping

Interaction

Error

\section{Sum of}

squares

603.4

20.7

23.4

8369.3

$$
4.2
$$

Spring

12

11

12.9

4.2

5.2

12.8

19.8

1.8

Summer
Unswept

23.0

3.1
$4 \quad 18$
Degrees of Mean

freedom square

1

603.4

20.7

23.4

0.11

0.7368

Tail

probability

0.0931

1

1

41

204.1 
Table 3. Results of 2-way analysis of variance of commercial-residential TSP differences $\left(\mu \mathrm{g} / \mathrm{m}^{3}\right)$.

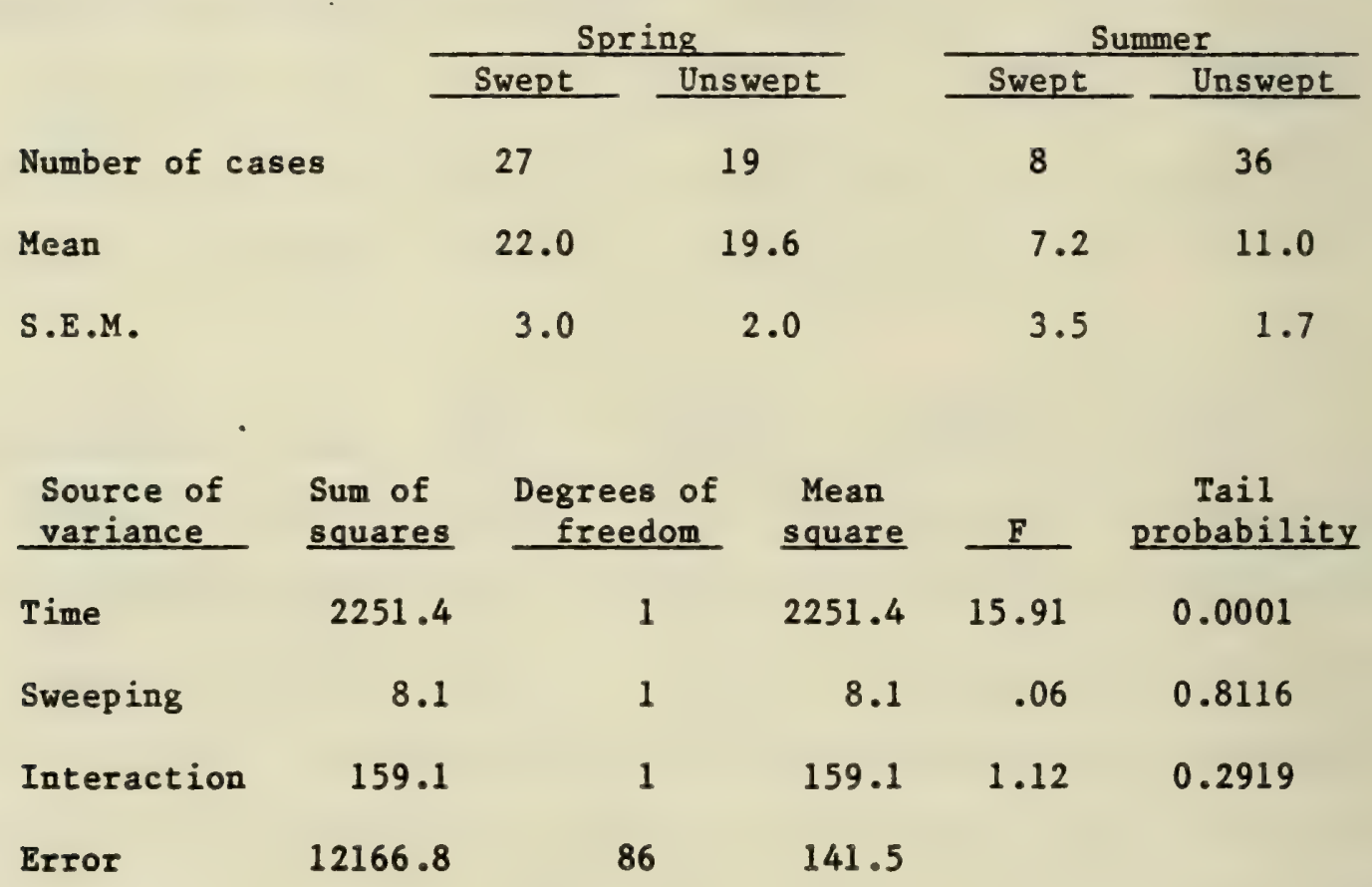


higher in spring than in summer in this case), but differences between swept and unswept periods look small. The analysis of variance table in the lower part of Table 3 confirms that the differences between swept and unswept periods were not significant, but also shows that seasonal differences were significant at the 0.0001 level. Interactions between sweeping and time were not significant in either comparison.

The main question addressed by this paper has now been answered. Although the test roadway was shown to be a source of TSP, no evidence was found to indicate that street sweeping had any effect, either beneficial or detrimental, on air quality at $7 \mathrm{~m}$ downwind.

Table 4 summarizes TSP concentrations and the two TSP differences discussed above. Mean TSP values are shown for three different data sets: (1) all samples, (2) the samples used in the downwind-upwind comparison, and (3) the samples used in the commercial-residential comparison. It is apparent that the mean TSP concentrations in the commercial (Mattis) and residential (John) sites do not vary much between sample sets. For example, the Mattis mean shows a maximum of 87 $\mu \mathrm{g} / \mathrm{m}^{3}$ and a minimum of $84 \mu \mathrm{g} / \mathrm{m}^{3}$ in the three data sets shown. Similarly, at the John site the highest mean was $65 \mu \mathrm{g} / \mathrm{m}^{3}$ and the lowest $62 \mu \mathrm{g} / \mathrm{m}^{3}$. Thus, regardless of the data set, mean TSP values were higher at both sites in spring than in summer. The Mattis site had higher concentrations by about $20 \mu \mathrm{g} / \mathrm{m}^{3}$ in the spring and about $8 \mu \mathrm{g} / \mathrm{m}^{3}$ in the 
Table 4. Summary of TSP concentrations and differences by season for three different sample sets.

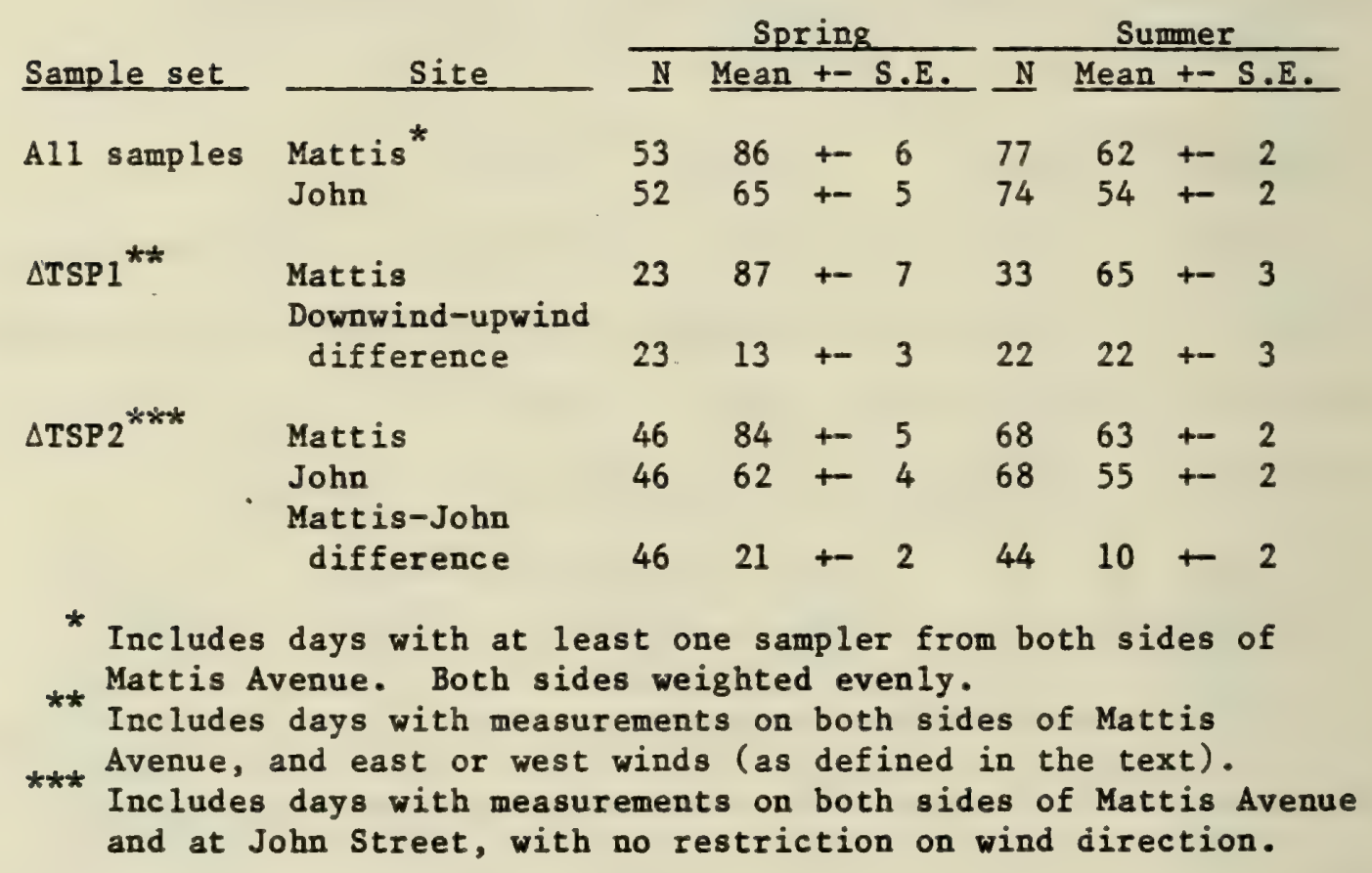


summer .

TSP concentration differences, however, display different seasonal behavior depending on the data set used. The mean commercial-residential difference $(\triangle T S P 2)$ was higher in spring than summer, as were TSP concentrations at both sites. The mean downwind-upwind difference ( $\triangle T S P 1$ ), on the other hand, was larger in the summer, when the Mattis site had lower concentrations.

The higher. TSP concentrations in spring are consistent with an agricultural soil dust source, which would be expected to be stronger in the spring from tilling operations, a lack of ground cover by crops, and generally stronger winds. It is reasonable for the Mattis site to have higher TSP concentrations than the John St. site because it is closer to the sources with prevailing winds, and because the John St. site is located in a somewhat older part of town, where the more mature trees may help to remove particles from the atmosphere.

The downwind-upwind differences were greatest in summer, when TSP concentrations were lower at both the John and Mattis sites. Others (e.g., Cowherd and Englehart, 1982) have found that roadway emissions depend on both traffic volume and roadway surface silt loading. We could find no evidence for significantly greater traffic volumes in 
summer than in spring, but according to data provided by Bender (1982), the mean and standard error of 11 spring measurements of total street loadings were $108 \pm 5 \mathrm{~g} /$ curb-m, while the same values for 10 summer measurements were $189 \pm 11 \mathrm{~g} /$ curb-m. Thus, the spring/summer ratio of loadings, 1.75 , is very close to that of $\triangle T S P 1,1.69$, and it appears that differences in street surface loadings between seasons could well have caused the observed seasonal differences in $\triangle T S P 1$.

\subsection{URBAN SOURCE APPORTIONMENT USING DICHOTOMOUS SAMPLER MEASUREMENTS}

\subsubsection{The Data}

The fine and coarse aerosol samples collected near Mattis Avenue were each analyzed for about 25 elements. Results, in the form of a summary of ambient concentrations, are given in Tables 5 and 6 . Maximum, minimum, and median concentrations are shown, along with means and their standard errors. The percentage of filters for which the various elements were present in less than detectable concentrations is also shown as an indication of the confidence we may have in the measurements for the various elements. For the purposes of these tables, less than detectable values were assumed to be one-half of the detection limits. Elements detected on $20 \%$ or less of the fine filters included $\mathrm{Cl}, \mathrm{Rb}, \mathrm{Sr}, \mathrm{Cd}, \mathrm{In}, \mathrm{Sn}$, and $\mathrm{Sb}$. Additional elements detected 
Table 5. Summary of ambient fine particle elemental concentrations measured on 95 sampling days, Mattis Ave., Champaign, Illinois, June - October, 1981.

\begin{tabular}{|c|c|c|c|c|c|c|}
\hline \multirow[b]{2}{*}{ Element } & \multirow{2}{*}{$\begin{array}{c}\% \text { of } \\
\text { filters } \\
<\text { D.L. }\end{array}$} & \multicolumn{5}{|c|}{ Concentration ${ }^{*}, \mathrm{ng} / \mathrm{m}^{3}$} \\
\hline & & Maximum & Median & Minimum & Mean & S.E.M. \\
\hline $\mathrm{Al}$ & 0 & 944 & 168 & 46 & 209 & 14.0 \\
\hline Si & 0 & 2308 & 283 & 98 & 366 & 32.6 \\
\hline $\mathbf{P}$ & 0 & 287 & 92 & 20 & 106 & 5.99 \\
\hline $\mathbf{s}$ & 0 & 7938 & 2187 & 356 & 2632 & 175 \\
\hline C1 & 100 & 12 & 4.0 & 2.0 & 4.8 & 0.25 \\
\hline $\mathrm{R}$ & 0 & 301 & 51 & 13 & 63.4 & 4.83 \\
\hline $\mathrm{Ca}$ & 0 & 285 & 45 & 13 & 52.6 & 4.06 \\
\hline $\mathrm{Ti}$ & 3 & 39 & 5.0 & 0.5 & 5.47 & 0.56 \\
\hline $\mathrm{v}$ & 53 & 4 & 1.0 & 0.5 & 1.18 & 0.07 \\
\hline$C_{r}$ & 39 & 15 & 2.0 & 0.5 & 3.60 & 0.34 \\
\hline Mn & 1 & 24 & 5.0 & 1.0 & 5.42 & 0.34 \\
\hline $\mathrm{Fe}$ & 0 & 306 & 61 & 17 & 72.2 & 5.30 \\
\hline $\mathrm{Ni}$ & 64 & 3 & 1.0 & 0.5 & 1.08 & 0.05 \\
\hline $\mathrm{Cu}$ & 10 & 30 & 3.0 & 0.5 & 3.70 & 0.37 \\
\hline $\mathrm{Zn}$ & 0 & 86 & 17 & 5.0 & 19.5 & 1.22 \\
\hline As & 79 & 7 & 2.5 & 1.5 & 2.75 & 0.13 \\
\hline $\mathrm{Se}$ & 47 & 4 & 1.0 & 0.5 & 1.46 & 0.08 \\
\hline $\mathrm{Br}$ & 0 & 64 & 21 & 7.0 & 25.9 & 1.49 \\
\hline $\mathrm{Rb}$ & 93 & 3 & 0.5 & 0.5 & 0.98 & 0.06 \\
\hline Sr & 94 & 3 & 1.0 & 0.5 & 1.19 & 0.06 \\
\hline Cd & 82 & 34 & 5.0 & 3.0 & 5.93 & 0.45 \\
\hline In & 96 & 10 & 4.0 & 3.0 & 4.74 & 0.18 \\
\hline Sn & 96 & 22 & 4.5 & 3.5 & 5.80 & 0.28 \\
\hline $\mathrm{Sb}$ & 90 & 24 & 8.0 & 6.0 & 9.94 & 0.42 \\
\hline $\mathrm{Pb}$ & 0 & 748 & 135 & 67 & 158 & 8.86 \\
\hline Total & & & & & & \\
\hline mass & - & 40000 & 15000 & 0 & 17032 & 774 \\
\hline
\end{tabular}

* Measurements less than detection limits were taken as one-half detection limits. 
Table 6. Summary of ambient coarse particle elemental concentrations measured on 95 sampling days, Mattis Ave., Champaign, Illinois, June - October, 1981.

\begin{tabular}{|c|c|c|c|c|c|c|}
\hline \multirow[b]{2}{*}{ Element } & \multirow{2}{*}{$\begin{array}{c}\% \text { of } \\
\text { filters } \\
\text { SD.L. }\end{array}$} & \multicolumn{5}{|c|}{ Concentration ${ }^{*}, \mathrm{ng} / \mathrm{m}^{3}$} \\
\hline & & Maximum & Median & Minimum & Mean & S.E.M. \\
\hline AI & 0 & $\overline{1730}$ & 574 & 98 & 619 & 33.2 \\
\hline $\mathrm{Si}$ & 0 & 6661 & 2306 & 432 & 2470 & 141 \\
\hline $\mathbf{P}$ & 0 & 80 & 52 & 19 & 51.1 & 1.51 \\
\hline $\mathrm{s}$ & 0 & 909 & 183 & 42 & 235 & 16.9 \\
\hline Cl & 0 & 340 & 34 & 6.0 & 41.6 & 4.31 \\
\hline $\mathrm{K}$ & 0 & 462 & 155 & 42 & 172 & 8.18 \\
\hline $\mathrm{Ca}$ & 0 & 5790 & 1197 & 330 & 1339 & 82.4 \\
\hline $\mathrm{Ti}$ & 0 & 108 & 38 & 8.0 & 42.2 & 2.31 \\
\hline $\mathrm{V}$ & 3 & 8 & 3.0 & 1.0 & 2.76 & 0.15 \\
\hline $\mathrm{Cr}$ & 21 & 12 & 4.0 & 0.5 & 4.40 & 0.33 \\
\hline Mn & 0 & 41 & 12 & 3.0 & 12.8 & 0.65 \\
\hline $\mathrm{Fe}$ & 0 & 968 & 373 & 85 & 408 & 18.5 \\
\hline $\mathrm{Ni}$ & 41 & 3 & 1.0 & 0.5 & 1.19 & 0.05 \\
\hline $\mathrm{Cu}$ & 10 & 8 & 2.0 & 0.5 & 2.50 & 0.14 \\
\hline $\mathrm{zn}$ & 0 & 50 & 17 & 3.0 & 18.0 & 0.84 \\
\hline As & 88 & 6 & 1.5 & 1.0 & 1.77 & 0.10 \\
\hline $\mathrm{Se}$ & 81 & 1 & 1.0 & 0.5 & 0.82 & 0.02 \\
\hline $\mathrm{Br}$ & 0 & 104 & 12 & 4.0 & 15.1 & 1.26 \\
\hline $\mathbf{R b}$ & 73 & 3 & 1.0 & 0.5 & 1.18 & 0.06 \\
\hline St & 15 & 10 & 3.0 & 0.5 & 3.05 & 0.18 \\
\hline $\mathrm{Cd}$ & 82 & 43 & 3.5 & 1.5 & 5.06 & 0.51 \\
\hline In & 81 & 13 & 5.0 & 2.5 & 5.21 & 0.23 \\
\hline $\mathrm{Sn}$ & 83 & 13 & 5.0 & 2.5 & 5.62 & 0.26 \\
\hline $\mathrm{Sb}$ & 85 & 27 & 7.5 & 3.5 & 9.38 & 0.47 \\
\hline $\mathrm{Pb}$ & 0 & 312 & 45 & 19 & 58.3 & 4.07 \\
\hline \multicolumn{7}{|l|}{ Total } \\
\hline mass & -- & 52000 & 18000 & 2000 & 19484 & 1118 \\
\hline
\end{tabular}

* Measurements less than detection limits were taken as one-half detection limits. 
on $50 \%$ or less of the filters were: $\nabla, \mathrm{Ni}$, and As. Thus, 15 elements were detected on more than half of the fine filters.

The elements As, Se, Cd, In, Sn, and Sb were detected on not more than $20 \%$ of the coarse filters, and in addition, $\mathrm{Rb}$ was detected on less than half of the coarse filters. This means that the remaining 18 elements were detected on at least half of the coarse particle filters.

As the earlier discussion of the method shows, data on source composition, as well as ambient concentrations of the elements, are necessary to apply the CEB method. Compositions of six sources considered likely to contribute to the measured ambient concentrations are given in Tables 7 and 8 , representing fine and coarse particles, respectively.

The compositions of urban street dust, rural soil, and dust from both limestone-covered and washed gravel-covered unpaved rural roads were measured, the ammonium sulfate composition is well known, and the composition of vehicle exhaust ("mobile") was taken from Dzubay et al, (1980). Both fine and coarse particle emissions of the latter two sources were assumed to have the same respective compositions, but separate fine and coarse compositions were measured for the other sources. 


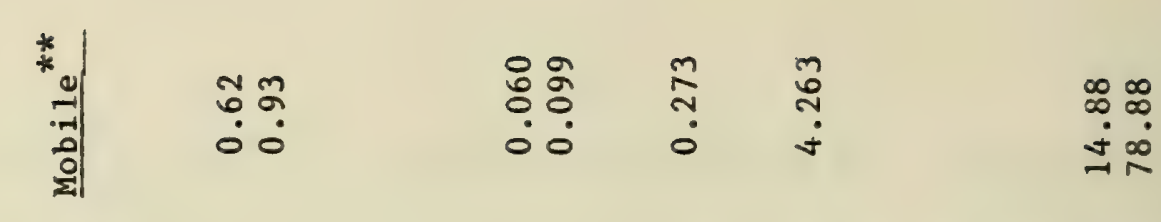

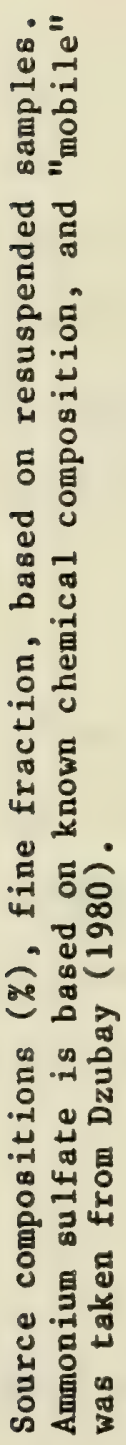

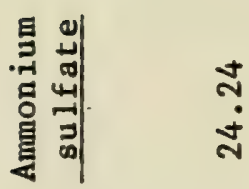

$\stackrel{\circ}{\stackrel{n}{n}}$

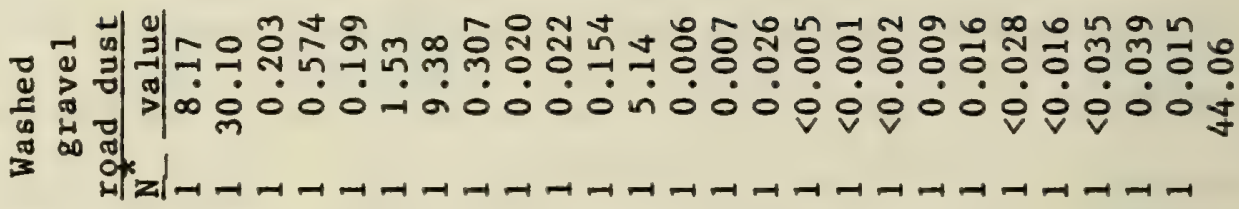

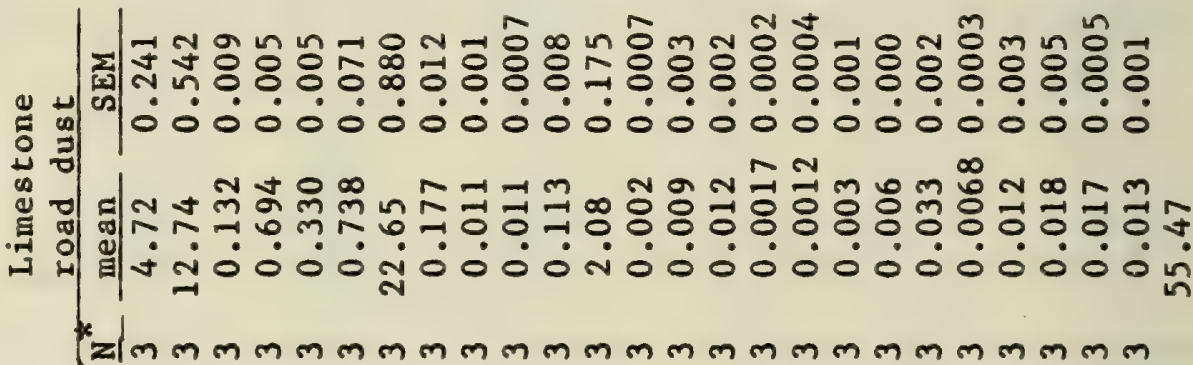

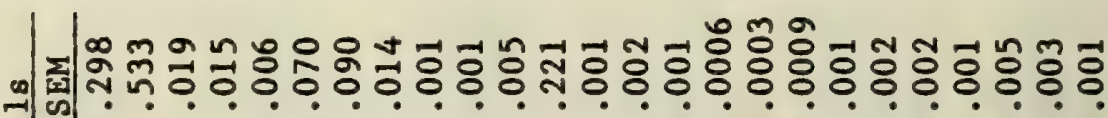
=|

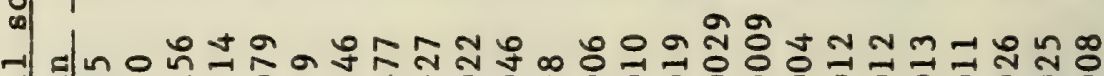

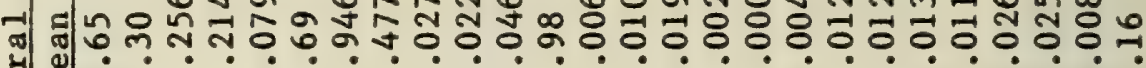
औ| Patenn

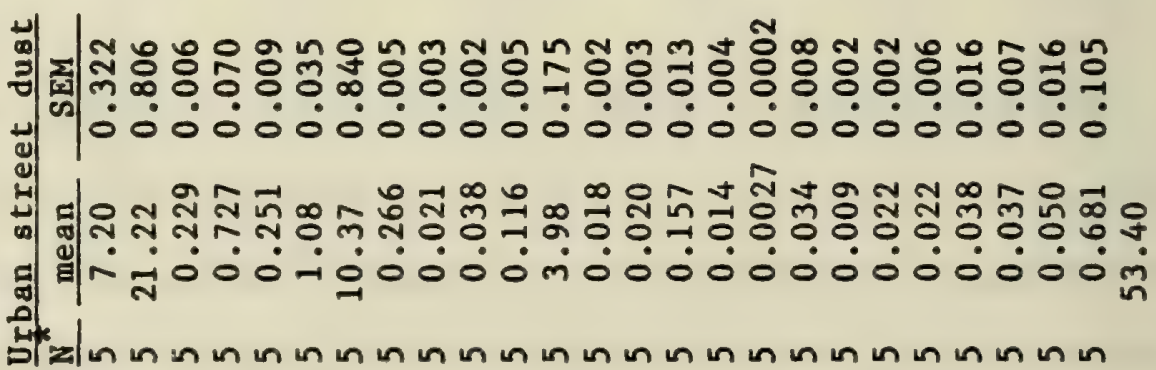

兽富

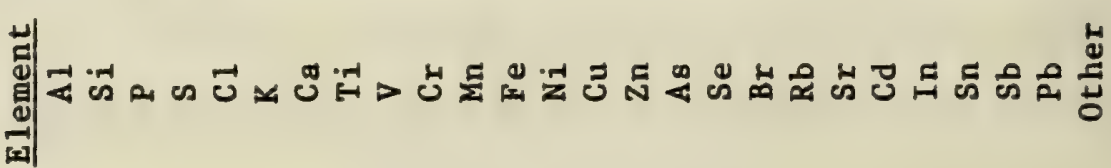




\begin{tabular}{|c|c|c|c|c|}
\hline \begin{tabular}{c|}
$*$ \\
$*$ \\
$*$ \\
-7 \\
-0 \\
0 \\
0
\end{tabular} & 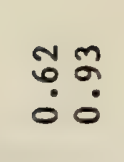 & $\begin{array}{l}\dot{0} \\
0 \\
0 \\
0\end{array}$ & ヘิ & ণ \\
\hline
\end{tabular}
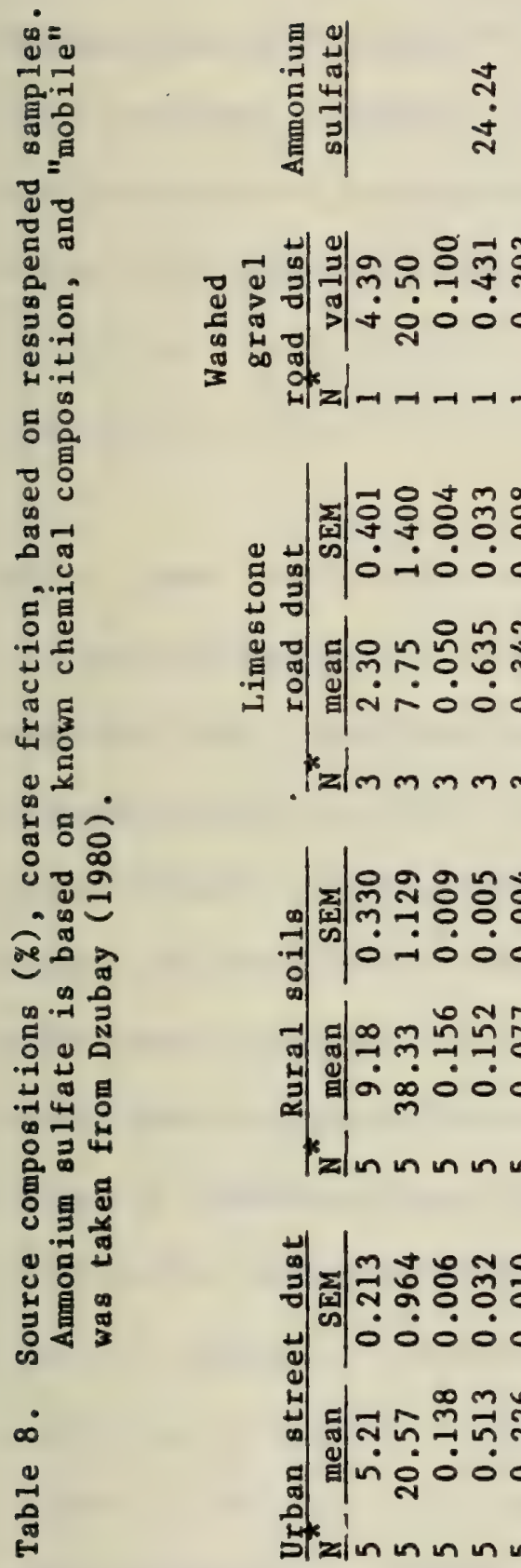

$$
\stackrel{0}{n}
$$

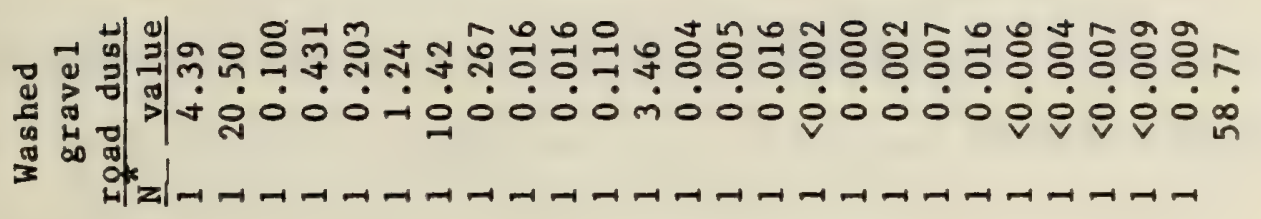
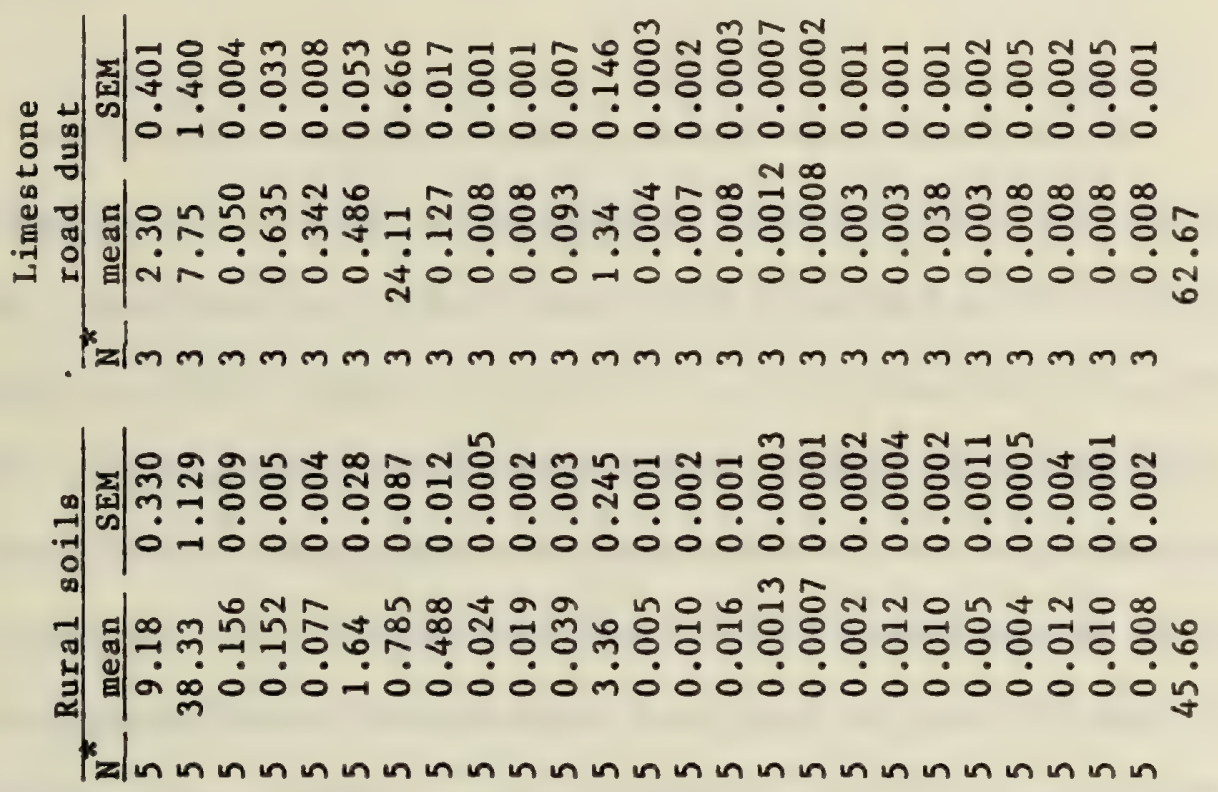

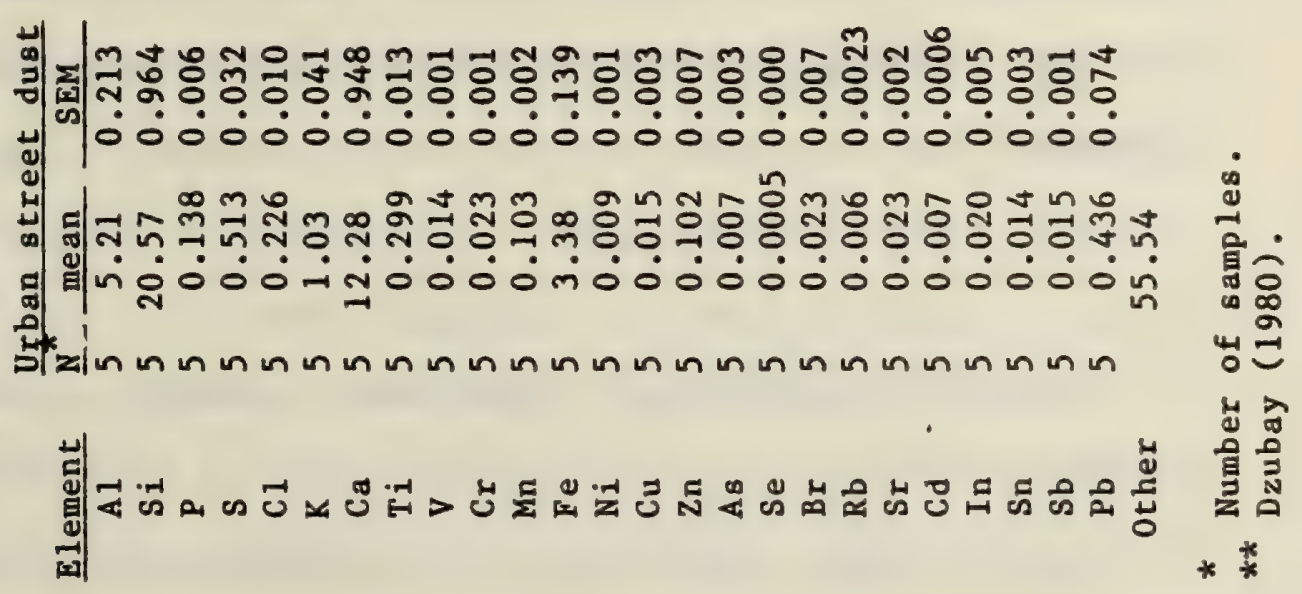


Samples of dry-sieved $(<53 \mu \mathrm{m})$ source materials were resuspended in a laboratory chamber and collected on separate filters in a dichotomous sampler closely matching that used for the ambient sampling. The resuspensions, sample collections, and analyses were performed by NEA, Inc., which also performed the $\mathrm{X}$-ray fluorescence analyses on the ambient samples.

The measured compositions shown in Tables 7 and 8 are mean values based on the number of samples shown for each of the various sources. Only a single sample of washed gravel dust was analyzed. For elements not detected in this single sample, values of one-half the detection limit were used for the source composition. This applies only to six elements in the fine fraction, and five in the coarse fraction, none of which was used in the least squares fitting. The standard errors given in the tables were used as the uncertainties on the source compositions needed as input data in Equation (4). Similarly, the standard errors of the mean ambient concentrations from Tables 5 and 6 were also used in Equation (4).

The variables that may be manipulated in search of a satisfactory CEB solution include 1) the number and nature of the sources considered, and 2) the elements used in the fit. In general, it appears that the fewer elements used, the better the fit. One must insure, bowever, that there are at least as many, and preferably one or two more, elements 
than sources, and that all significant sources of the elements are included among the sources considered. In the search for a satisfactory CEB solution, poor results were indicated by 1) any negative contributions to the aerosol mass, 2) total source contributions of more than $100 \%$ (both of which are physically impossible), and 3) large deviations of ratios of predicted and observed concentrations (larger/smaller) from 1.0.

The CEB method may be used in a number of ways to apportion source contributions from a set of ambient data. The usual method is to apply the technique to a data set consisting of the mean concentrations of each element. Alternatively, the technique can be applied separately to each individual sample, and the results presented in the form of histograms showing the distributions of percent contributions of the various sources identified. In this work, we first analyzed a data set of mean concentrations. Once the main sources were identified, we applied the technique to each individual sample, assuming that the same set of sources identified for the mean was active for each individual sample also. This was was somewhat of a simplification, since it ignored sources that may have been important on some individual days, but contributed only negligibly to the mean concentrations. However, the results serve to show the kind of sample-to-sample variability that can occur in source contributions to total aerosol mass from day to day. 
5.3.2 CEB on Mean Ambient Concentrations

Results of a series of attempts to fit the mean fine particle data are given in Table 9. Six trials were attempted before a satisfactory solution was reached. The first trial used 13 elements in the fit and al1 six potential sources:

1. urban street dust ("street" in the table),

2. local rural soil ("soil"),

3. limestone unpaved road dust ("1imestone"),

4. dust from unpaved roads covered with washed glacially-derived gravel ("washed gravel"),

5. ammonium sulfate ("sulfate'), and

6. vehicle exhaust ("mobile").

Compositions of fine particles derived from these sources were given in Table 7 .

Trial 1 was obviously less than satisfactory because calculated contributions of three of the six sources considered were negative.

Trial 2 used the same six sources, but a shorter list of fitting elements: $\mathrm{Al}, \mathrm{Si}, \mathrm{S}, \mathrm{K}, \mathrm{Ca}, \mathrm{Fe}, \mathrm{Br}$, and $\mathrm{Pb}$. Results were somewhat better, because only two of the calculated contributions were negative, 


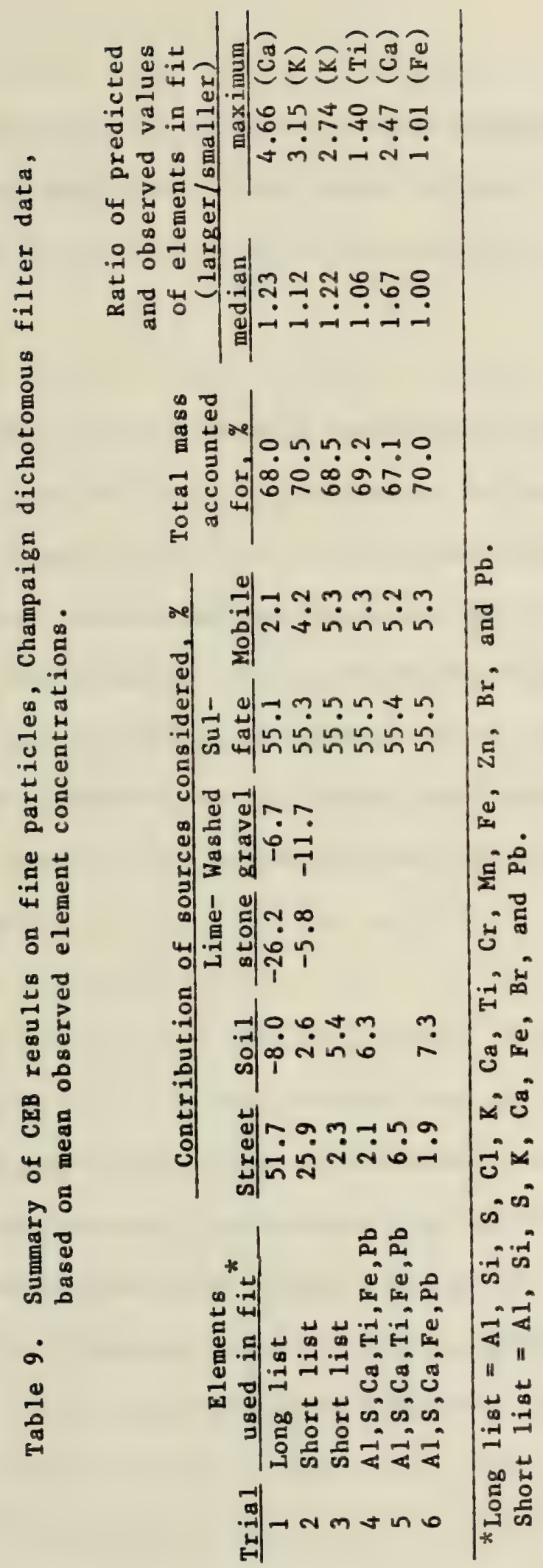


and the median and maximum ratios of predicted and observed values were smaller, but the results were still unsatisfactory because of the negative contributions calculated for limestone and washed gravel.

Trial 3 kept the same short list of fitting elements, but eliminated limestone and washed gravel as potential sources, as suggested by the results of the first two trials. This time there were no negative contributions, but the median and maximum ratios were still unacceptably high. Close examination of the results showed that the $\mathrm{Si}, \mathrm{K}$, and $\mathrm{Br}$ ratios were the largest; that is, predicted concentrations of these elements, based on a least squares fit of the data, showed the largest deviations from observed concentrations.

Thus, in Trial 4, the four sources were kept the same as in Trial 3, but $\mathrm{Si}, \mathrm{K}$, and $\mathrm{Br}$ were removed from the list of elements used in the fit. The calculated contributions were little changed from the previous trial, but the ratios were considerably improved, the median being 1.06 and the maximum 1.40 (for $T i$ ). This suggested removing $T i$ from the elements in the fit, but first we wanted to see the results of eliminating another potential source, namely soil.

Thus, Trial 5 was carried out with the same fitting elements, but soil was not considered as a potential source. The results in Table 9 
show that elimination of soil as a source was not desirable. No negative contributtions occurred, but the median and maximum ratios increased dramatically from the results of Trial 4.

Having learned that soil was a necessary source, we proceeded to carry out another trial without $\mathrm{Ti}$ as a fitting element, in hopes of improving on the previous results. The results of Trial 6, shown in Table 9, were excellent. No negative contributions appeared, the total contributions of all the sources considered did not exceed $100 \%$, and the maximum ratio of observed and predicted values was 1.01 . This best fit of the observed concentrations to the potential sources indicates that urban street dust contributed $1.9 \%$ of the total fine particle mass, rural soils $7.3 \%$, ammonium sulfate $55.5 \%$, and vehicle exhaust $5.3 \%$. The remaining $30 \%$ was not explained, but is likely to consist largely of water, nitrates, and organic matter, which are not measured in our analyses .

The detailed results of Trial 6 on the fine particle means are given in Table 10. The upper part of the table gives the results for the elements used in the least squares fit, and the lower part gives the results for the remaining elements. The table shows the calculated ("predicted") contribution of each element from each source, and the sums of all contributions ("predicted" total concentrations), as well as the corresponding observed concentrations. The rightmost column gives 
Table 10. Chemical element balance for Champaign dichotomous fine particles.

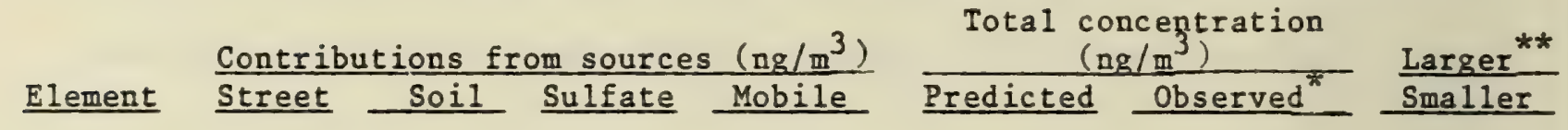
Elements used in fitting

$\begin{array}{lrrrr}\mathrm{Al} & 27 & 181 & 0 & 0 \\ \mathrm{~S} & 3 & 3 & 2620 & 6 \\ \mathrm{Ca} & 39 & 13 & 0 & 0 \\ \mathrm{Fe} & 15 & 57 & 0 & 1 \\ \mathrm{~Pb} & 3 & 0 & 0 & 155\end{array}$

\begin{tabular}{rrrr}
208 & $209+-$ & 14 & 1.00 \\
2632 & $2632+-$ & 175 & 1.00 \\
53 & $53+-$ & 4 & 1.00 \\
73 & $72+-$ & 5 & 1.01 \\
158 & $158+-$ & 9 & 1.00 \\
\cline { 3 - 3 } & & Median & $=1.00$
\end{tabular}

Remaining elements

$\begin{array}{lrrr}\mathrm{Si} & 80 & & 632 \\ \mathrm{P} & 1 & \\ \mathrm{C} 1 & 1 & \\ \mathrm{~K} & 4 & \\ \mathrm{Ti} & 1 & & \\ \mathrm{~V} & 0 & & \\ \mathrm{Cr} & 0 & \end{array}$

Mn $\quad 0 \quad 1$

$\mathrm{Ni} \quad 0 \quad 0$

$\mathrm{Cu} \quad 0 \quad 0$

Zn $\quad 1 \quad 0$

As $\quad 0 \quad 0$

Se $\quad 0 \quad 0$

$\begin{array}{lll}\text { Br } & 0 & 0\end{array}$

$\mathrm{Rb} \quad 0 \quad 0$

Sr $\quad 0 \quad 0$

Cd 00

In 0

Sn $\quad 0 \quad 0$

$\begin{array}{lll}\mathrm{Sb} & 0 & 0\end{array}$

Other $201 \quad 501$

032

40

$1 \quad 0$

4

7
0


the ratio of the larger of the predicted and observed concentrations to the smaller. For the elements used in the fit, the results in Table 10 show excellent agreement between the predicted and observed concentrations: i.e., ratios near 1.00 . Of the remaining elements, a few were overpredicted, i.e., $\mathrm{P} / \mathrm{O}>0$, but most were underpredicted, i.e., $0 / P>0$. Underprediction was expected for many of these elements, since many of them are emitted by sources not considered in this analysis.

Continuing with results of the CEB analysis of mean concentrations, we discuss next the various trials in the analysis of the coarse particles; these results are summarized in Table 11.

Decisions regarding specific sources to consider in the various CEB trials on the coarse particle means were guided by the principle that sources having similar composition are difficult to resolve reliably (Gordon, 1980). Since we perceived that urban vehicle-raised dust would likely be an important aerosol source, and that other likely sources of aerosol, such as vehicle exhaust and soil dust, were also known to be sources of the street dust (Hopke et al, 1980), so that the compositions of the street dust might be quite similar to several other sources. Thus, it was not clear whether a soil source, for example, could be considered at the same time as a street dust source, or not. The following discussion illustrates this concern. 

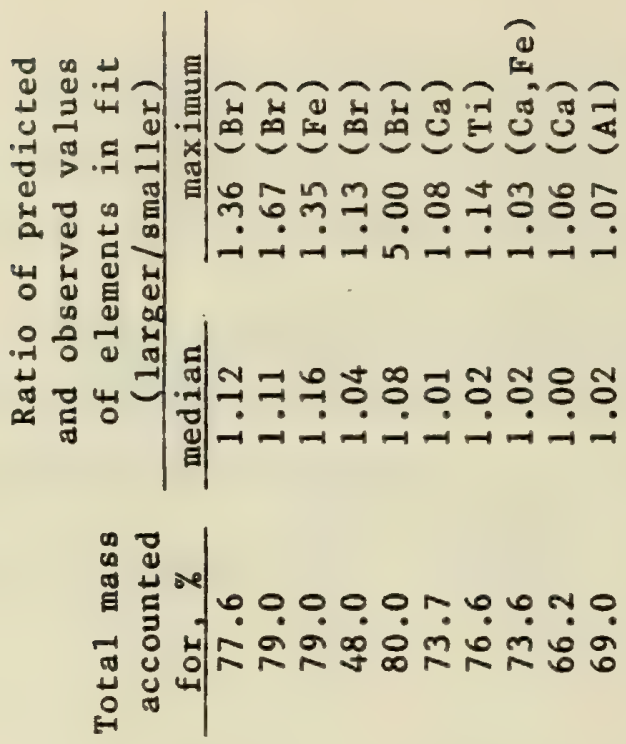

章

ग̊

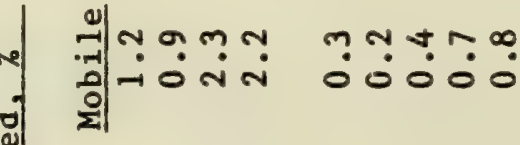

o.

a 4

-

논

ค.

- U

$\infty$

하

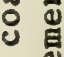

둥

a

$\pm>$

可

40

电

3 บ

4

> 뮹

40

总

苞

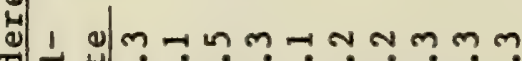
क

대

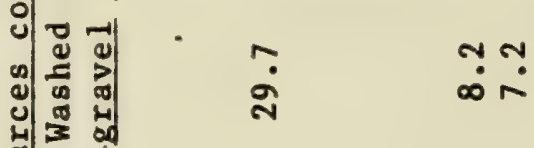

อิ

4ु)

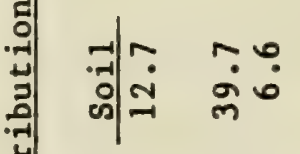

क ?

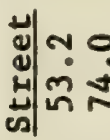

a.

령

$m$

ฝี $\backsim$

ஸं

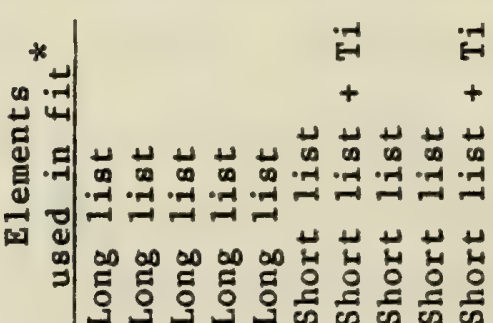

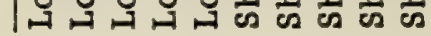

- $\begin{gathered}-4 \\ \text { H. }\end{gathered}$ 
Results of ten successive CEB trials are given in Table 11. The first five trials used $\mathrm{Al}, \mathrm{Si}, \mathrm{S}, \mathrm{K}, \mathrm{Ca}, \mathrm{Fe}, \mathrm{Br}$, and $\mathrm{Pb}$ in the least squares $\mathrm{fit}$, and the last five used only $\mathrm{Al}, \mathrm{S}, \mathrm{Ca}, \mathrm{Fe}$, and $\mathrm{Pb}$, and in two cases, also Ti. The table refers to these element groups as the "long" and "short" lists, respectively, but note that the lists are not the same as those given the same labels in the analysis of the fine particles (Table 9).

Trial 1 considered five sources: urban street dust, soil dust, limestone dust, sulfate, and vehicle exhaust. The calculated mass contributions were all positive, and together accounted for $77.6 \%$ of the total observed mass. The median ratio of predicted and observed concentrations (larger/smaller) for the elements used in the fit was 1.12 , and the maximum ratio was 1.36 for $\mathrm{Br}$. This was a reasonably good fit for the first trial, but of course it was necessary to try a number of other combinations of sources to see which would give the best fit.

Trial 2 attempted a fit using only street dust, soil, and mobile sources, and the same fitting elements as in the previous trial. Slightly more mass was accounted for overall, and the median ratio decreased slightly, but the maximum ratio (still for $\mathrm{Br}$ ) increased to 1.67 . 
Trials 3 and 4 were carried out to see if the urban street dust source could effectively be replaced with a combination of soil and unpaved road sources. Trial 3 used soil and limestone sources along with sulfate and mobile, and Trial 4 added a washed gravel source as we11. The results in Table 11 show that Trial 3 maintained the $79.0 \%$ overall mass explained in the previous trial, and achieved median and maximum ratios of 1.16 and 1.35 , respectively, whereas Trial 4 achieved ratios even closer to 1.0 , but accounted for only $48 \%$ of the total aerosol mass.

Trial 5, the last with the long list of fitting elements, was very similar to Trial 2, but was an attempt to see whether the mobile source could be eliminated as long as street dust was included. Trial 5 accounted for $80 \%$ of the total mass, the highest of all the trials, and achieved a respectable mean ratio of 1.08 , but its maximum ratio jumped to 5.00 , for $\mathrm{Br}$.

Trial 6, the first to use the short list of fitting elements, was essentially a repeat of Trial 2 with different fitting elements. It accounted for $73.7 \%$ of the total mass, and obtained excellent ratios of 1.01 and 1.08. Trial 7 was essentially a repeat of Trial 6 with Ti added to the fitting elements. Its results were very similar, but $\mathrm{Ti}$ was responsible for the maximum ratio of 1.14 . 
Thus, Ti was dropped from the list of fitting elements in Trial 8 . At this point it seemed quite obvious that street dust, sulfate, and mobile sources were all required to give a good fit to the ambient observations, and it only remained to determine whether any better fit could be achieved by adding one or more of the soil and road dust sources. Trial 8 added soil, and obtained excellent results, accounting for $73.6 \%$ of the total mass, with median and maximum ratios of 1.01 and 1.03 , respectively.

Trial 9 added washed gravel road dust, rather than soil, and also obtained satisfactory results, although the total mass accounted for decreased slightly, from $73.6 \%$ to $66.2 \%$. Trial 10 included both soil and washed gravel. It accounted for $69 \%$ of the total mass, and yielded median and maximum ratios of 1.02 and 1.07, respectively. ( $\mathrm{Ti}$ was added to the list of fitting elements so that there would be more elements than sources.)

Other combinations of sources were tried, but all involved the combination of urban street dust and limestone dust, and results gave negative contributions for one or more of the sources.

Thus, Trials 8,9 , and 10 all gave reasonable fits to the observed mean coarse particle concentrations. Quite likely the reason for the 
several possible combinations of sources is that soil and washed gravel have rather similar compositions, so that it is difficult to distinguish between them. Based on slightly better overall results, in terms of the fraction of the mass accounted for, and the ratios of predicted and observed concentrations, we chose Trial 8 as the one for which to show detailed results of the CEB on the coarse means. These detailed results are given in Table 12, but the results in Table 11 make it clear that where the soil source is listed in Table 12, it represents some combination of soil and washed gravel road dust.

Table 11 shows that whenever street dust was included among the various sources considered, it contributed the majority of the coarse particle mass. This is illustrated in Table 12, which gives the detailed results of Trial 8. Street dust accounted for $66.0 \%$ of the total coarse particle mass, whereas soil, sulfate, and mobile sources contribute less than $8 \%$ among them. This heavy contribution from street dust is also reflected in the contributions to the individual elements, except, of course, for $S$, which came mostly from sulfate aerosol. Even $\mathrm{Pb}$, which is generally acknowledged as a tracer for vehicle exhaust, came predominanty from the street source for the coarse particles.

Again, the ratios indicate that an excellent fit to the ambient mean coarse concentrations was achieved using the five elements indicated. As was the case for the fine particles, the remaining elements were 
Table 12. Chemical element balance for Champaign dichotomous coarse particles.

Element $\underline{\text { Contributions from sources }\left(\mathrm{ng} / \mathrm{m}^{3}\right)} \frac{\left(\mathrm{ng} / \mathrm{m}^{3}\right)}{\text { Street Soil Sulfate Mobile }} \frac{\text { Predicted Observed }}{\text { Smaller }}$

Total concentration

Elements used in fitting

$\begin{array}{lrrrr}\mathrm{Al} & 585 & 46 & 0 & 0 \\ \mathrm{~S} & 58 & 1 & 177 & 0 \\ \mathrm{Ca} & 1380 & 4 & 0 & 0 \\ \mathrm{Fe} & 380 & 17 & 0 & 0 \\ \mathrm{~Pb} & 49 & 0 & 0 & 9\end{array}$

Remaining elements

$\begin{array}{lrr}\mathrm{Si} & 2311 & \\ \mathrm{P} & 16 & \\ \mathrm{C} 1 & 25 & \\ \mathrm{~K} & 116 & \\ \mathrm{Ti} & 34 & \\ \mathrm{~V} & 2 & \\ \mathrm{Cr} & 3 & \\ \mathrm{Mn} & 12 & \\ \mathrm{Ni} & 1 & \\ \mathrm{Cu} & 2 & \\ \mathrm{Zn} & 11 & \\ \mathrm{As} & 1 & \\ \mathrm{Se} & 0 \\ \mathrm{Br} & 3 & \\ \mathrm{Rb} & 1 & \\ \mathrm{Sr} & 3 & \\ \mathrm{Cd} & 1 & \\ \mathrm{In} & 2 & \\ \mathrm{Sn} & 2 & \\ \mathrm{Sb} & 2\end{array}$

Other $6240 \quad 229$

Total $11236 \quad 501$

\% Contrib 66.0
1920

10

$0 \quad 0$

$8 \quad 0$

20

$0 \quad 0$

$0 \quad 0$

$0 \quad 0$

$0 \quad 0$

$0 \quad 0$

$0 \quad 0$

$0 \quad 0$

$0 \quad 0$

$0 \quad 0$

$0 \quad 0$

$0 \quad 0$

$0 \quad 0$

$0 \quad 0$

$0 \quad 0$

$29 \quad 552$

$501 \quad 728$

2.9
$0 \quad 0$

0
0
0
0
9

631
235
1384
397
58

2503

16

26

124

36

2

3

12

1

2

12

1

0

5

1

3

1

2

2

7070

49

62

$12640 \quad 17030$

$$
\begin{aligned}
& 620+-33 \quad 1.02 \\
& 235+17 \quad 1.00 \\
& 1339+821.03 \\
& 408+19 \quad 1.03 \\
& 58+-4 \frac{1.00}{\text { Median }}
\end{aligned}
$$

$$
\begin{array}{rrr}
2470+- & 141 & 1.01 \\
51+- & 2 & 3.18 \\
42+- & 4 & 1.62 \\
172+- & 8 & 1.39 \\
42+- & 2 & 1.17 \\
3+- & 0 & 1.50 \\
4+- & 0 & 1.33 \\
13+ & 1 & 1.08 \\
1+- & 0 & 1.00 \\
3+ & 0 & 1.50 \\
18+- & 1 & 1.50 \\
2+ & 0 & 2.00 \\
1+- & 0 & - \\
15+- & 1 & 3.00 \\
1+- & 0 & 1.00 \\
3+ & 0 & 1.00 \\
5+- & 1 & 5.00 \\
5+ & 0 & 2.50 \\
6+- & 0 & 3.00 \\
9+ & 0 & 4.50
\end{array}
$$

73.6 
quite consistently underpredicted.

A graphical illustration of the overall results on both fine and coarse particles is given in Figure 6. This illustrates the overall dominance of sulfate as the major fine particle source, and street dust as the major coarse particle source in Champaign in the summer and fall.

It is instructive to examine the observed concentrations of the individual elements in relation to the concentrations predicted in the various trials, i.e., when considering various combination of potential sources. In this way, insights were provided regarding the necessity to include specific sources, and possibilities of missing sources or questionable source compositions were suggested.

Table 13 provides a summary of the major sources of individual elements in both fine and coarse particles. It also summarizes the fit achieved for the element--that is, whether the predicted and observed concentrations agreed closely, the predicted concentration was greater than that observed (overprediction), or the predicted concentration was less than that observed (underprediction). 


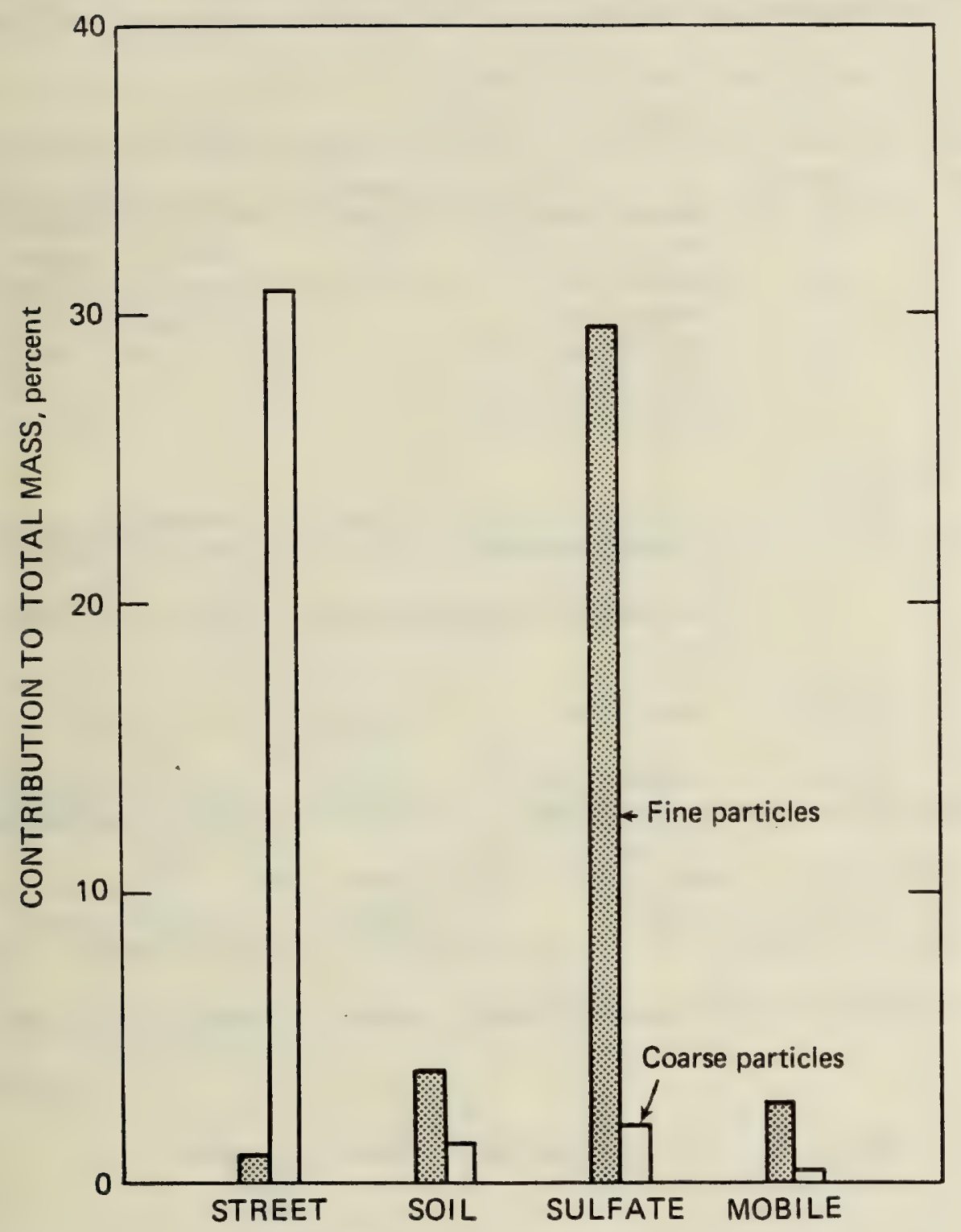

Figure 6. Summary of source contributions to total aerosol mass. 
Table 13. Summary of major sources and fit characteristics of individual elements in fine and coarse particle classes.

Fine

\begin{tabular}{|c|c|}
\hline $\begin{array}{c}\text { Main } \\
\text { source }\end{array}$ & Fit \\
\hline Soil & $\begin{array}{l}\text { Excellent if } \\
\text { neither } \mathrm{Si} \text { nor } \\
\mathrm{Ti} \text { in fit. } \\
\text { Otherwise, } \\
\text { underpredicted: } \\
\text { o/P }=1.1-1.2 \\
\text { (with soil in } \\
\text { fit). }\end{array}$ \\
\hline
\end{tabular}

Coarse

\begin{tabular}{|c|c|}
\hline $\begin{array}{l}\text { Main } \\
\text { source }\end{array}$ & Fit \\
\hline Street & $\begin{array}{l}\text { Good if Si not } \\
\text { in fit. Other- } \\
\text { wise, overpre- } \\
\text { dicted. P/O = } \\
1.1-1.2 \text {. }\end{array}$ \\
\hline
\end{tabular}
Si Soil Overpredicted. Street Overpredicted. If used in fit, If used in fit, $\mathrm{P} / 0=1.5$; otherwise, $\mathrm{P} / 0=$ $\mathrm{P} / 0=1.08$; otherwise, $\mathrm{P} / 0=$ 2.0 1.02 .

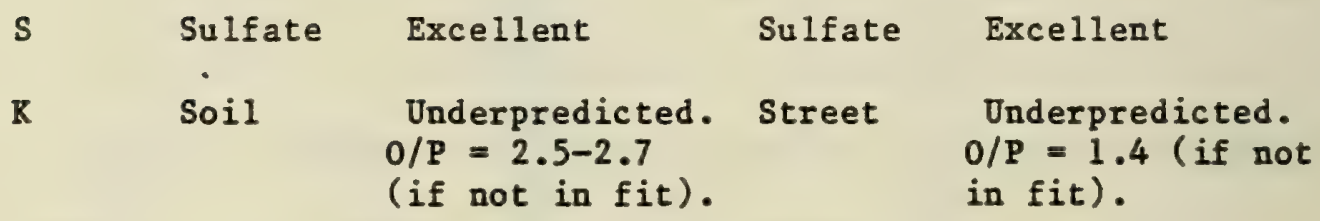
Ca Street Good
Street
Slightly over- predicted.
Ti
Soil
Overpredicted.
Street
Underpredicted. $\mathrm{P} / 0=1.6$. $0 / P=1.2$.

\begin{tabular}{|c|c|c|c|c|}
\hline Mn & $\begin{array}{l}\text { Soil, } \\
\text { mobile }\end{array}$ & $\begin{array}{l}\text { Underpredicted. } \\
0 / \mathbf{P}=2.5\end{array}$ & Street & Good \\
\hline $\mathrm{Fe}$ & Soil & Good & Street & Good \\
\hline $\mathrm{Zn}$ & Mobile & $\begin{array}{l}\text { Substantially } \\
\text { underpredicted. } \\
0 / P=4.8\end{array}$ & Street & $\begin{array}{l}\text { Underpredicted. } \\
0 / \mathrm{P}=1.5\end{array}$ \\
\hline $\mathrm{Br}$ & Mobile & $\begin{array}{l}\text { Overpredicted. } \\
\mathrm{P} / 0=1.7\end{array}$ & $\begin{array}{l}\text { Street, } \\
\text { mobile }\end{array}$ & $\begin{array}{l}\text { Underpredicted. } \\
0 / P=3.0\end{array}$ \\
\hline ob & Mobile & Good & Street & Good \\
\hline
\end{tabular}


Aluminum Based on Tables 7 and $9, A 1$ in fine particles came primarily from soil dust, whereas coarse particle Al came from street dust. Success in predicting Al concentrations was closely related to which elements were used in the fit. Fine Al was predicted very well if neither Si nor $\mathrm{Ti}$ was among the elements used in the fit. If either or both of those elements were used in fitting, then fine Al was underpredicted.

Similarly, coarse Al was predicted well if $\mathrm{Si}$ was not used in the fit, but was slightly overpredicted if it was.

Silicon Table 10 shows that fine $\mathrm{Si}$ also came predominantly from the soil, with some contribution from street dust. When used as a fitting element, fine $\mathrm{Si}$ was overpredicted by a factor of about 1.5. If not among the fitting elements, fine $\mathrm{Si}$ was overpredicted by a factor of about 2.0 .

Coarse Si came mostly from street dust, as Tables 9 and 10 indicate. Again, coarse $\mathrm{Si}$ was overpredicted, with the amount of the overprediction varying according to whether $\mathrm{Si}$ was used in the fit or not. 
In fine particles, Al underprediction occurred with $\mathrm{Si}$ in the fit because $\mathrm{Si}$ was higher in abundance with respect to $\mathrm{Al}(\mathrm{Si} / \mathrm{Al}=3.50$, data from Table 7) in the main source (soil) than it was in the atmosphere $(\mathrm{Si} / \mathrm{Al}=1.75$, data from Table 5).

Thus, the best fit to both elements was a compromise on a source contribution that made the predicted Si higher than observed, and AI less than observed.

Thus, if the fine Al abundances in the sources are correct, then the fine $\mathrm{Si}$ abundances in one or more of the sources must be too high, since the prediction based on the source composition gave more $\mathrm{Si}$ than actually observed. On the other hand, if the fine $\mathrm{Si}$ abundances are correct, then either the fine Al abundances are too low, or else we are overlooking a significant source of $\mathrm{Al}$.

In the coarse particles, $A 1$ was overpredicted with $S i$ in the fit because Si/Al was slightly smaller (3.95) in the main source (street dust) than it was in the atmospheric coarse particles (3.99).

These considerations emphasize that proper characterization of sources, in terms of the composition of their aerosol, is very 
important. That is, we must know and use the element abundances in the dust that blows off the soil or the street, not simply the abundances in bulk soil or street dust. To generate samples of aerosol, bulk samples were resuspended before being sampled and analyzed. Thus, we hope we have measured the proper material. However, $\mathrm{Si}$ in soils is thought to occur both in relatively large particles as quartz, and in relatively small particles as both quartz and clay minerals, while Al would occur primarily in clay minerals in Illinois soils.

In the environment the clay minerals are found agglomerated into, or attached to, relatively large particles, but during saltation it is the relatively small clay-sized particles that become airborne and are small enough to remain airborne for long times (hours).

Thus, it is likely that nature discriminates against the large-particle $\mathrm{Si}$ in favor of the small-particle Al and $\mathrm{Si}$, through gravitational deposition of the large particles soon after they enter the atmosphere. This is essentially the argument Rahn (1976) used to explain atmospheric $\mathrm{Si} / \mathrm{Al}$ ratios lower than those of bulk crustal materials. The crucial question is whether the resuspension process used to prepare the samples of fine and coarse source materials is adequate to simulate the natural resuspension processes. 
Sulfur Ammonium sulfate was the dominant source of $S$ in both fine and coarse particles, and the fit was always exact.

Potassium As indicated in Table 13, fine $\mathrm{K}$ came mainly from the soil, and street dust was the main source of coarse $R$. Both fine and coarse $R$ were underpredicted by sizable amounts, although the underprediction was somewhat less if $\mathrm{K}$ was used in the fit. It appears that there must be additional sources of both fine and coarse $k$ that we have not identified.

Caicium Both fine and coarse $\mathrm{Ca}$ came mainly from urban street dust, although soils also made an important contribution to fine $\mathrm{Ca}$. Predicted and observed concentrations agreed closely for fine $\mathrm{Ca}$, unless soil dust was not among the sources, in which case Ca was overpredicted by a factor of 2.5. Thus, it appears that soil, with its relatively low abundance of $\mathrm{Ca}$, is necessary for an overall good fit of the fine data. Coarse Ca was consistently overpredicted, but only slightly, unless soil was omitted as a source, in which case the overprediction was larger.

Titanium Soil dust was the main source of fine $\mathrm{Ti}$, and street dust contributed most of the coarse $\mathrm{Ti}$. Fine $\mathrm{Ti}$ was overpredicted by a factor of 1.6 , while coarse $\mathrm{Ti}$ was underpredicted, with an $0 / \mathrm{P}$ ratio of 1.2 . 
Manganese Fine Mn was contributed about equally by soil and vehicles, but additional sources are needed to fully account for the observed fine Mn, since the CEB yielded an underprediction. The coarse Mn fit was good, with street dust the main source.

Iron Soil dust was the major source of fine Fe, and street dust the major source of coarse Fe, and predicted and observed concentrations were in close agreement for both size classes. Omitting soil from the fine particle sources and street dust from the coarse-particle sources resulted in sizable underpredictions, and confirmed the need for these sources to explain the respective observations.

Zinc A vehicular source was the major one identified for fine $\mathrm{Zn}$, but the substantial underprediction indicates that there must have been others. Coarse $\mathrm{Zn}$ was also underpredicted, although not as much as the fine $\mathrm{Zn}$, and street dust was the major source.

Bromine and Lead We consider these two elements together, since the ultimate source of both of them in Champaign is exhaust from vehicles burning leaded fuels. Street dust also contains both elements, since a significant fraction of tailpipe exhaust is deposited on road surfaces, but $\mathrm{Br}$ is greatly depleted in the street dust, relative to $\mathrm{Pb}$. The $\mathrm{Pb} / \mathrm{Br}$ ratio assumed for both fine and coarse vehicle exhaust (Dzubay 
et al, 1980) was 3.49 , while the ratios measured in fine and coarse street dust were 20.0 and 19.0 , respectively.

Fine particle $\mathrm{Br}$ was overpredicted by a factor of 1.7 , whether $\mathrm{Br}$ was used in the fit or not. Since Br compounds are relatively volatile, the overprediction suggests losses of $\mathrm{Br}$ from the aerosol during aging, so that the observed concentration of $\mathrm{Br}$ would be disproportionately low relative to $\mathrm{Pb}$.

Fine $\mathrm{Pb}$ came overwhelmingly from vehicle exhaust, and was fit very we11.

Additional insights into the behavior of $\mathrm{Br}$ and $\mathrm{Pb}$ came from the CEB results on the mean ambient composition of the coarse particles. When both $\mathrm{Br}$ and $\mathrm{Pb}$ were used in the fit, the results depended on which sources were considered. If both mobile and street dust sources were considered, $\mathrm{Br}$ was underpredicted and $\mathrm{Pb}$ was overpredicted. For $\mathrm{Br}$ the $0 / \mathrm{P}$ ratio was 1.36 and 1.67 in two such trials. For $\mathrm{Pb}$ in the same trials, the $\mathrm{P} / \mathrm{O}$ ratio was 1.22 and 1.34 .

In the case where only the street dust source was included in the fit, $\mathrm{Pb}$ was slightly underpredicted $(0 / \mathrm{P}=1.04)$, but $\mathrm{Br}$ was greatly 
underpredicted $(0 / P=5.00)$, indicating that the $\mathrm{Pb}$ abundance in the street dust was quite reasonable in relation to the other elements used in the fit, but that there was far too little $\mathrm{Br}$ in the street dust to account for what was in the atmosphere.

On the other hand, if only the mobile source was included in the fit, the $\mathrm{Pb}$ was predicted exactly $(\mathrm{P} / \mathrm{O}=1.00)$, and the $\mathrm{Br}$ was slightly overpredicted $(P / 0=1.13)$.

If only $\mathrm{Pb}$ was used in the fit, and both street dust and mobile sources of coarse particles were considered, the $\mathrm{Pb}$ was predicted exactly, but the $\mathrm{Br}$ was seriously underpredicted $(0 / \mathrm{P}=3.00,3.75$, and 3.00 in three trials). These results reflect the dominance of the relatively Br-deficient street dust as a coarse particle source, but if they are valid, they do not explain all the observed coarse Br.

Remaining Elements Many of the remaining elements occurred in concentrations that were less than detectable more than half of the time. Results for these elements must therefore be considered very tentative. The poorly-detected elements in the fine fraction (Table 5) include $\mathrm{Cl}, \nabla, \mathrm{Ni}, \mathrm{As}, \mathrm{Rb}, \mathrm{Cd}, \mathrm{In}, \mathrm{Sn}$, and $\mathrm{Sb}$. The remaining elements not on this list were consistently underpredicted, an indication that their sources are not well known, or at least not accounted for in this 
analysis.

Poorly-detected elements in the coarse fraction (Table 6) include As, $S e, R b, C d, I n, S n$, and $S b$. Of the coarse elements not on this list, there were several for which predicted values were within $10 \%$, or $1 \mathrm{ng} / \mathrm{m}^{3}$, of the observed value. These were $\mathrm{V}, \mathrm{Cr}, \mathrm{Mn}, \mathrm{Ni}, \mathrm{Cu}$ (except when the street source was omitted), and Sr. The other elements not on the poor $1 y$-detected 1 ist were consistent $1 y$ underpredicted; these include $\mathrm{P}, \mathrm{Cl}$ and $\mathrm{Zn}$.

\subsubsection{CEBs on Individual Samples}

CEB analysis was applied to individual fine and coarse samples, using the same source compositions as were used for the analyses of the mean compositions, and using the same sets of sources identified in the earlier analyses of the mean compositions. That is, since street dust, soil dust, sulfate, and mobile sources were identified as the relevant sources for both fine and coarse particles from the CEB analyses of the respective mean compositions, the same sources were assumed to be valid for the CEB analyses of the individual samples. 
Results of the CEB analyses on individual fine filter data are shown in Figure 7 in the form of histograms of the frequency of occurrence of the percent contributions of the four sources to the total fine aerosol. There is an overall qualitative agreement between these results and those based on the mean fine concentrations. That is, the histograms show that sulfate accounted for $50-60 \%$ of the fine mass in a typical case, while street dust, soil, and mobile sources typically contributed less than $10 \%$. This agrees with the results obtained from the mean concentrations (Table 10), in which sulfate accounted for $55.5 \%$; soil, 7.3\%; mobile, $5.3 \%$; and street dust, $1.9 \%$ of the total fine mass. Note that none of the CEB calculations on individual filter yielded negative contributions of any of the sources. Two samples indicated sulfate contributions over $100 \%$, however.

Results of the CEB analyses on the individual coarse filters are shown in Figure 8. Again, there was good qualitative agreement with the results based on the mean concentrations. The figure shows that street dust typically contributed $60-80 \%$ of the coarse mass, in good agreement with the figure of $66.0 \%$ obtained from the means. The figure also shows typical contributions of less than $10 \%$ from soil dust, sulfate, and mobile sources, and these agree with the values of $5.9 \%$, $4.3 \%$, and $0.4 \%$ obtained by analysis of the mean concentrations. Note also, however, that the analyses of the individual filters yielded several street dust contributions over $100 \%$, and frequent negative contributions of soil and mobile sources. The cause of these negative 


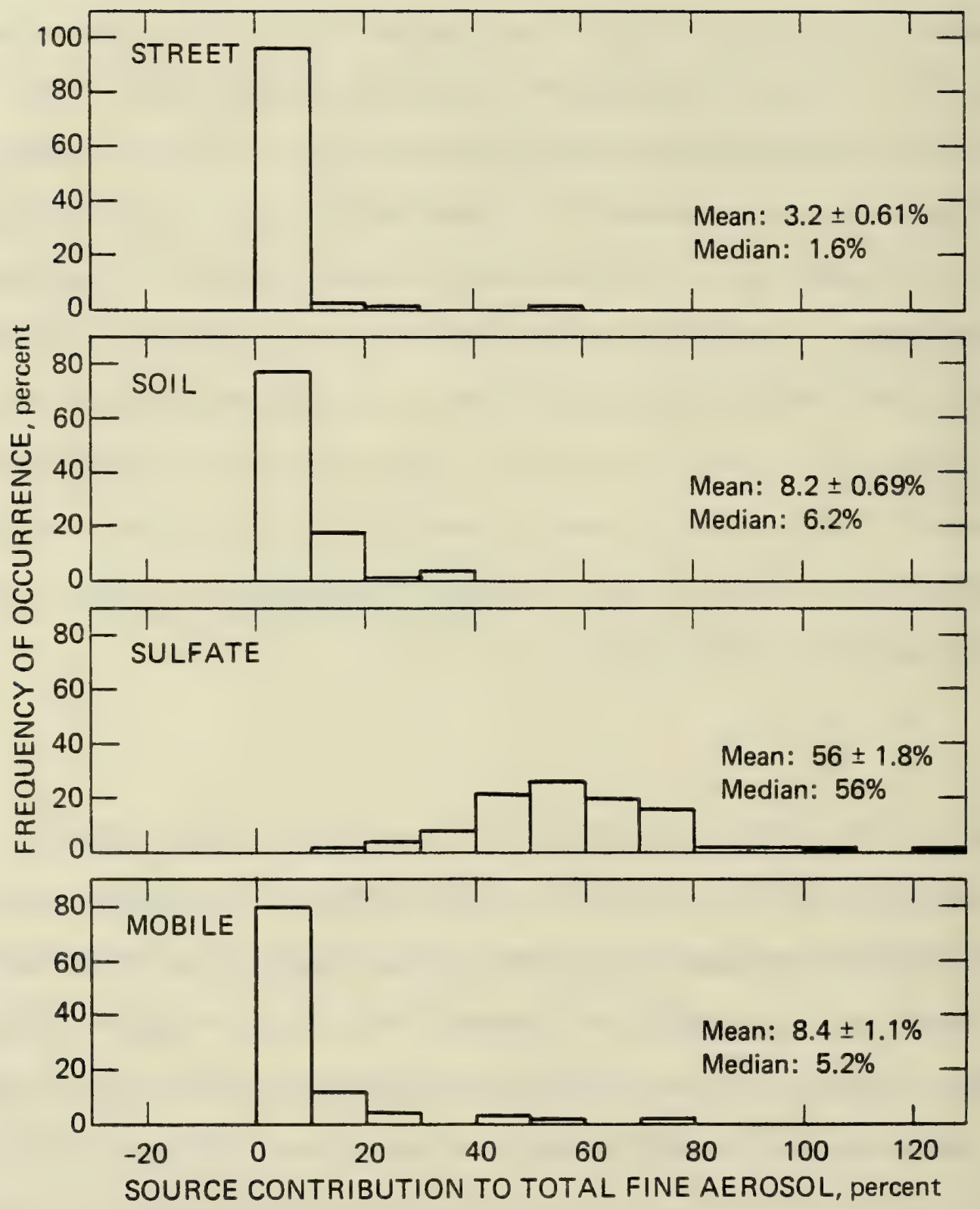

Figure 7. Frequency distributions of source contributions, based on CEB analyses of individual samples -- fine aerosol. 


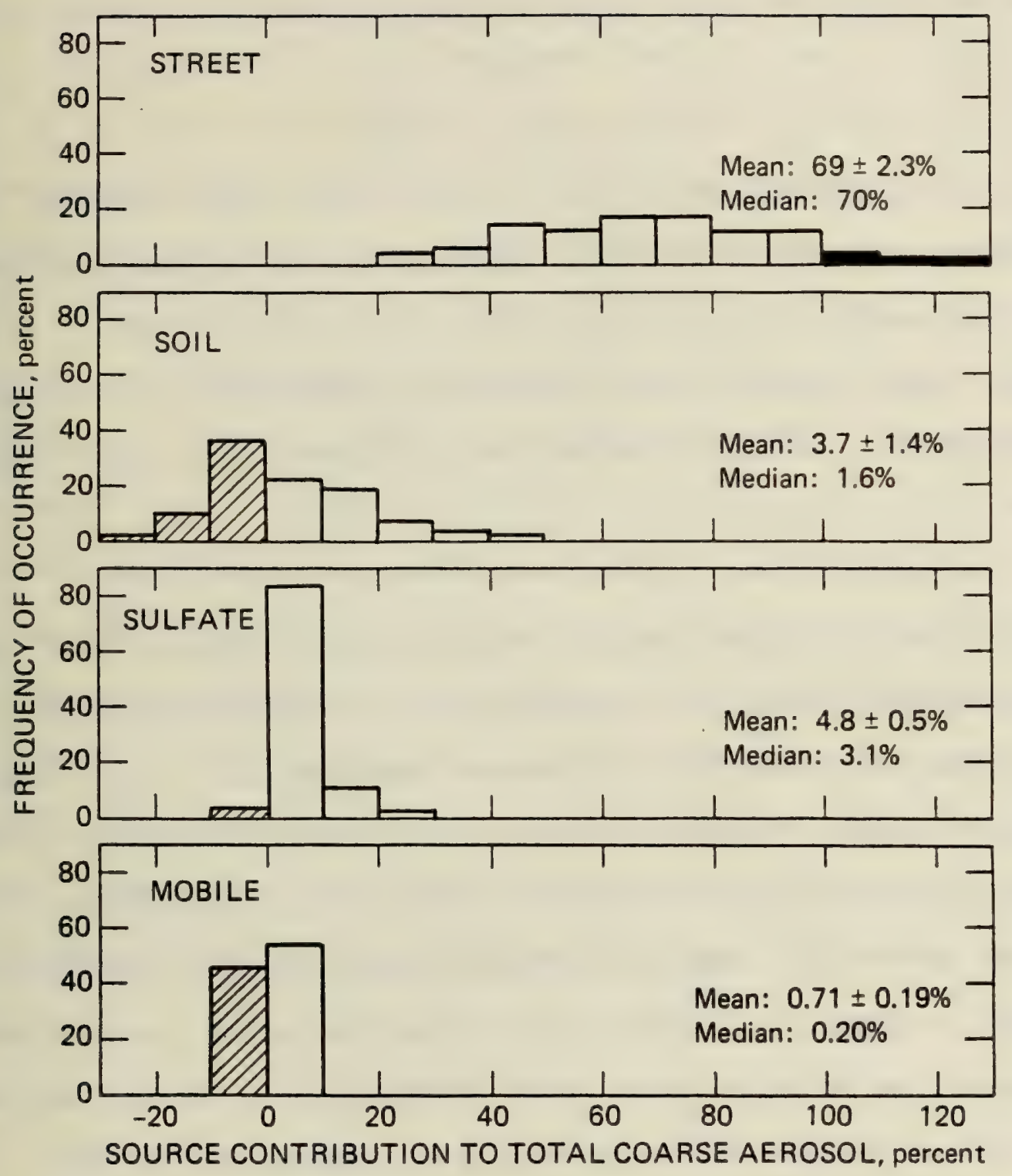

Figure 8. Frequency distributions of source contributions, based on CEB analyses of individual samples -- coarse aerosol. 
contributions is not clear, but it is possibie that they may reflect the occasional presence of other sources not considered in the analyses, or they may simply be random errors on true contributions very near zero.

One may ask how the results of the source apportionment, which indicate that street dust contributed about $2 \%$ of the fine mass and $66 \%$ of the coarse mass, agree or disagree with the hivol filter results, which showed that Mattis Ave increased the downwind TSP concentration by about $22 \mathrm{\mu g} / \mathrm{m}^{3}$ when the wind was blowing perpendicular to the roadway.

To compare these results, we first must express the source apportionment results in terms of the street dust contribution to the total aerosol, rather than its separate contributions to the fine and coarse aerosol. Tables 5 and 6 give the mean concentrations of total fine and coarse aerosol as 19.5 and $17.0 \mu \mathrm{g} / \mathrm{m}^{3}$, respectively., or a total of $35.6 \mathrm{\mu g} / \mathrm{m}^{3}$. Thus, the fine and coarse aerosol accounted for $53 \%$ and $47 \%$, respectively, of the total aerosol sampled by the dichotomous sampler. Weighting the street dust contributions to the fine and coarse aerosols by $53 \%$ and $47 \%$, respectively, and summing, we see that street dust contributed about $32 \%$ of the total mass sampled by the dichotomous sampler, i.e., the mass on particles smaller than $15 \mu \mathrm{m}$ diameter. 32\% of the mean total mass $\left(35.6 \mu \mathrm{g} / \mathrm{m}^{3}\right)$ is $11.7 \mu \mathrm{g} / \mathrm{m}^{3}$. It is important to note that the street dust source that contributed $32 \%$ to the particle mass less than $15 \mu \mathrm{m}$ may include, but is not necessarily limited to, 
that from nearby Mattis Ave.

It is also important to be clear about the hivol results. First of a11, it is important to know the range of particle diameters collected by the hivol samplers. Unfortunately, the evidence on this point is not as clear as one would like. Kolak and Visalli (1981) mentioned that hivols collect particles up to about $100 \mu \mathrm{m}$, but gave neither a reference nor an efficiency of collection for $100 \mu \mathrm{m}$ particles. Sierra Instrument Co. product literature says that hivols collect "over a wide size range, up to 30-100 $\mu \mathrm{m} . "$ Wedding et al., found in wind tunnel tests that hivols collected particles of about $19 \mathrm{um}$ with $50 \%$ efficiency when the angle of incidence of the wind to the collector was 45 degrees. At 0 degrees, particles of $11 \mu \mathrm{m}$ diameter were collected with $50 \%$ efficiency. For both wind angles, particles larger than those mentioned were collected with smaller (but not zero) efficiencies. Thus, it appears likely that the standard hivol samplers collected particles larger than $15 \mu \mathrm{m}$ with greater efficiency than the dichotomous sampler did.

Figure 4 and the discussion of it indicate that, overall (i.e., in both east and west winds), Mattis Ave added $21.7 \mu g / m^{3}$ to the downwind concentration of total mass in the size range sampled by the hivols. Previous discussion (p. 44) made clear, however, that in "west" winds, i.e., those between 203 and 338 degrees, only about $9 \mu \mathrm{g} / \mathrm{m}^{3}$, or about 
$11 \%$ of the mean hivol TSP for such cases, was added to the downwind concentration. The overall mean TSP for Mattis Ave, equally weighting both east and west sides of the street, was $71.1 \mathrm{\mu g} / \mathrm{m}^{3}$, almost exactly double that of the dichotomous sampler, but the dichotomous sampler collected particles having diameters in the range $0-15 \mu \mathrm{m}$, whereas the hivol collected numerous particles $>15 \mu \mathrm{m}$ in diameter. Thus, it is clear that about half of the mean hivol TSP concentration came from particles $>15 \mu \mathrm{m}$ in diameter.

Now, if $32 \%$ of the total mass between 0 and $15 \mu \mathrm{m}$ was street dust, and $66 \%$ of the coarse mass $(2.5-15 \mu \mathrm{m})$ was street dust, and we assume that the street dust contribution $(66 \%)$ to the coarse mass also holds for the $>15 \mu \mathrm{m}$ size range, then the weighted average percent contribution of steet dust to the hivol TSP would be $(32 \% \times 0.5)+(66 \%$ $x \quad 0.5)=49 \%$. This should be true on the average, over all wind directions, but since we know that Mattis Ave contributed $11 \%$ of the mean total hivol mass in west winds, we might expect that the street dust contribution to the hivol ISP would be even greater than $49 \%$ in west winds.

Thus, in west winds, at least half of the hivol TSP mass should be steet dust, and Mattis Ave contributed only $11 \%$, so other streets farther upwind must have contributed the rest. 
If this is so, the relative influence of Mattis Ave on the street dust contribution to the $0-15 \mu \mathrm{m}$ size class (dichotomous data) should have been small also, since a local source would be expected to contribute more mass in the larger particle sizes than the smaller ones. That is, the street dust contribution to the $0-15 \mu \mathrm{m}$ mass with west winds should not have been noticeably different from that in other wind directions, nor should it have been a strong function of the fraction of time during a given sample that the wind was from the westerly sector.

The data plotted in Figures 9 and 10 agree with these hypotheses. Figure 9 shows the street dust contribution to the $0-15 \mu \mathrm{m}$ mass as a function of wird direction. It is true that the single value in 248 293 degree subsector was unusually large, but the mean values for the southwest and northwest subsectors, which had 22 and 8 samples, respectively, showed no real differences from most other directions. The mean value for the east wind subsector was also large, but again, based on only 3 samples.

Figure 10 shows the street dust contribution to the total $0-15 \mu \mathrm{m}$ mass as a function of the fraction of the time winds were from the westerly sector during sample collection. The street dust contribution clearly did not increase with an increasing fraction of westerly winds, and may, on the contrary, have decreased. 


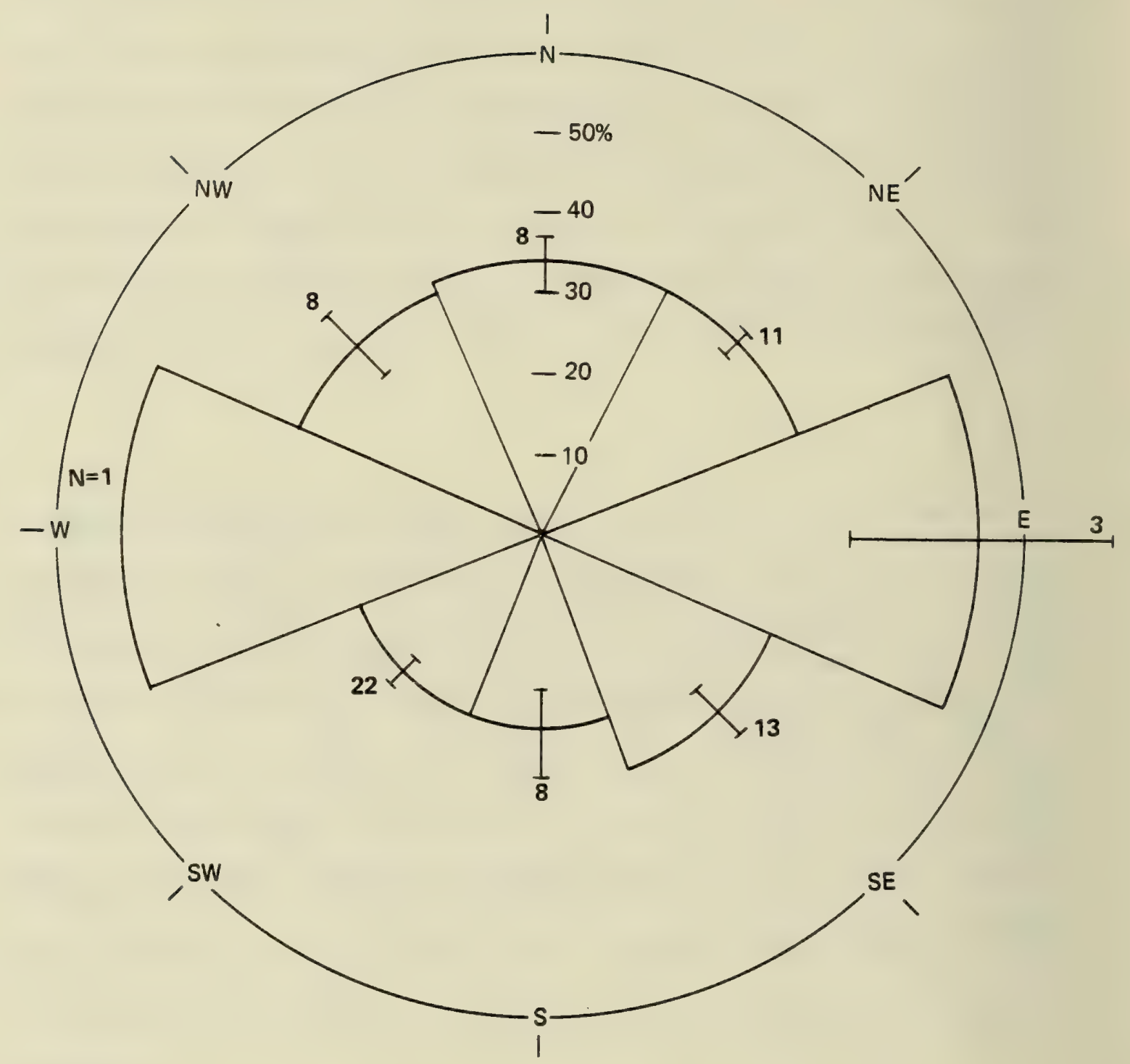

Figure 9. Mean street dust contributions to total mass $<15 \mu \mathrm{m}$ as a function of wind direction. Radial bars are plus/minus 1 S.E.M. $N$ = number of samples in each direction category. 


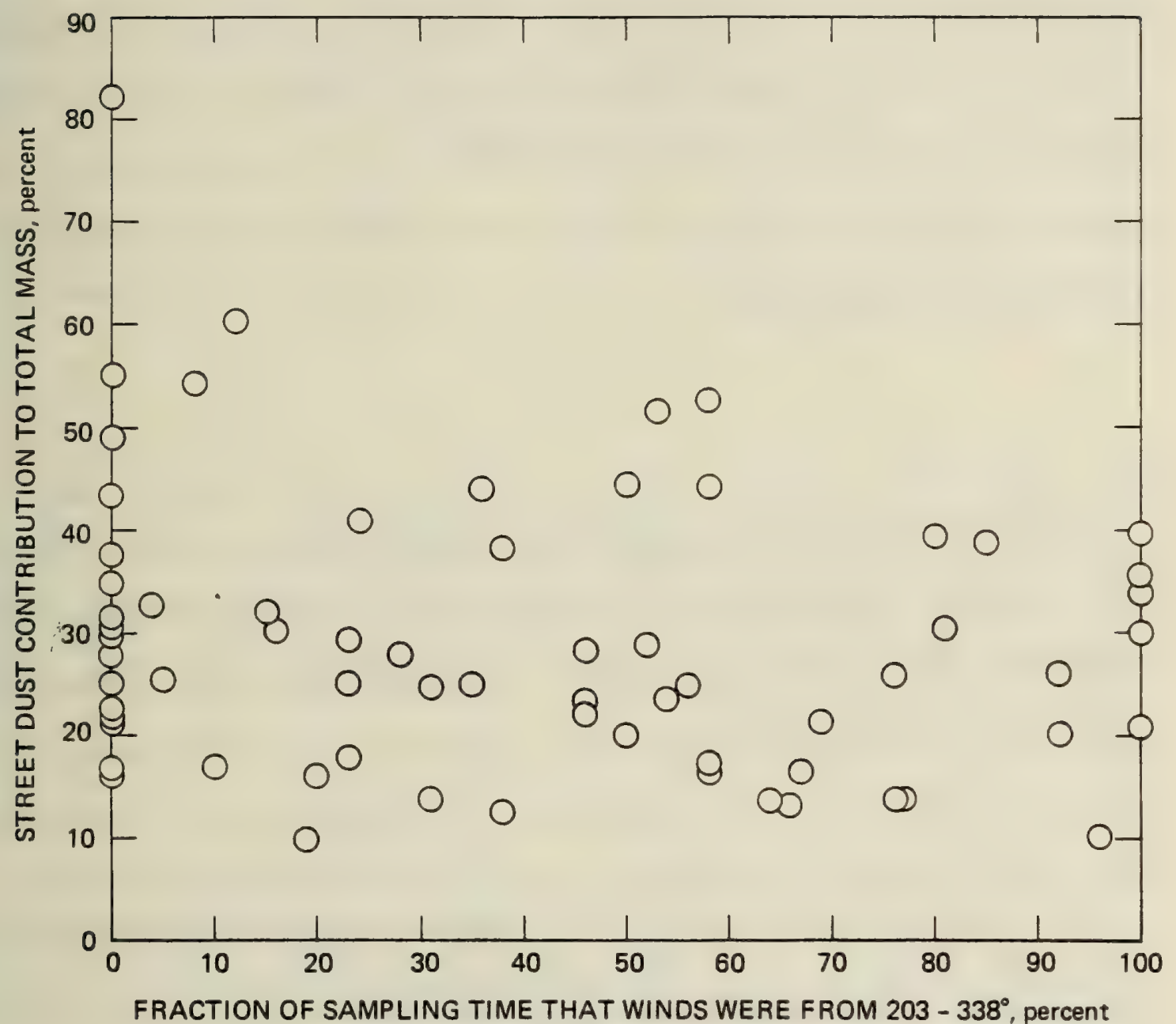

Figure 10. Street dust contribution to total mass $<15 \mu \mathrm{m}$ as a function of the fraction of time winds were from the westerly sector during sample collection. 
Thus, the two results are consistent. Mattis Ave contributed 11\% (mostly particles with diameters larger than $15 \mu \mathrm{m}$, apparently) to the total hivol mass in westerly winds, but the overall street dust contribution to the hivol TSP was approximately $50 \%$. The Mattis Ave contribution to the $0-15 \mu \mathrm{m}$ mass was not detectable, but overall, street dust accounted for $32 \%$ of the $0-15 \mu$ m mass.

6 SUMMARY AND CONCLUSIONS

Inhalable particle mass concentrations were on the average $75 \%$ of the standard TSP in a rural area, and 50\% of the standard TSP in Champaign. These values agree well with previous rural and urban measurements, respectively.

This study was carried out 1) to compare mass concentrations of inhalable particles (i.e., those with aerodynamic diameters of $15 \mu \mathrm{m}$ or less) with TSP concentrations measured in standard hivol samplers, 2) to assess the effects of street sweeping on urban TSP concentrations, and 3) to determine the sources of airborne particulate matter in Champaign, and their relative contributions to total aerosol mass. 
Comparison of 98 pairs of standard and size-selective $(<15 \mu \mathrm{m})$ hivol filters collected in a rusal agricultural area indicated that inhalable particle mass was about $75 \%$ of the standard TSP concentration, on the average. Comparison of mean total mass concentrations from 95 fine and coarse pairs of dichotomous samples and 236 hivol TSP concentrations in an urban area showed that urban IP concentrations averaged about $50 \%$ of standard TSP concentrations. These results agree closely with previously published results of both urban and rural comparisons.

To determine the effects of street sweeping on urban TSP concentrations, three standard hivol samplers were operated near a four lane commercial roadway, and an additional sampler was operated in a residential area of Champaign, Illinois, between April and October, 1981. When winds were blowing across the test roadway in either direction, concentrations at 7 m downwind from the curb exceeded upwind by an average of about $22 \mu \mathrm{g} / \mathrm{m}^{3}$. In a comparison of downwind-upwind TSP concentration differences during a period of regular weekly street sweeping with those from another period when sweeping was discontinued, analysis of variance was unable to detect a significant effect of sweeping on the amount of TSP produced by the roadway. Thus, for a commercial urban roadway clearly shown to be a source of airborne particles, street sweeping could not be shown to have any effect in reducing the amount of dust produced. 
Application of a chemical element balance model to apportion sources of f $\mathrm{ne}$ and coarse particles collected in a dichotomous virtual impactor indicated that street dust accounted for about $2 \%$ of the fine mass and $66 \%$ of the coarse mass, on the average, or about $32 \%$ of the total particle mass $<15 \mu \mathrm{m}$. If the street dust contribution to the coarse particles also applies to larger particles collected by the standard hivol samplers, street dust sources should account for about $50 \%$ of the hivol mass.

Although street dust accounted for $32 \%$ of the mean mass of particles $<15 \mu \mathrm{m}$, the influence of nearby Mattis Ave on the composition of both fine and coarse particles was negligible. Thus, it appears that the increases in TSP observed in the hivol samplers downwind of Mattis Ave were predominantly particles $>15 \mu \mathrm{m}$. On the other hand, $32 \%$ of the mass $<15 \mu \mathrm{m}$ was contributed by street dust, mostly in the $2.5-15 \mu \mathrm{m}$ size range, and apparently from streets more distant than Mattis Ave.

\section{ACRNOWLEDGEMENTS}

This research was carried out under support from the Illinois Department of Energy and Natural Resources, Project 10.093. Mr. William P. Murphy was the Project Manager. 
Sample collection was facilitated by the efforts of Bruce Komadina, who devised a convenient means of mounting the hivols and supervised their installation. Thanks are also due Randall $K$. Stahlhut, for programming assistance, Eberhard Brieschke, who helped with the installations, and Mr. Kenneth Porter, Mr. Lawrence Boastick, and Dr. and Mrs. Glenn Stout, who allowed us to operate samplers on their property. The Illinois EPA, through the assistance of Mr. Arden Ahne11, Mr. Bob Hutton, and Mr. John Shrock, provided, calibrated, and maintained the standard hivols, and provided and weighed the filters. We also thank Mr. Michael Terstriep and Mr. Michael Bender for their cooperation in supplying data. 


\section{REFERENCES}

Alpert, D. J., and P. K. Hopke, 1980: A quantitative determination of sources in the Boston urban aerosol. Atmos. Environ., 14, 1137-1146.

Arnold, E., and R. G. Draftz, 1979: Identification of sources causing TSP non-attainment. Document No. 79/21, Illinois Institute of Natural Resources, Chicago.

Arnold, E., R. G. Draftz, and D. Kolaz, 1980: Identification of sources causing TSP non-attainment in the Peoria area. Document No. $80 / 09$, Illinois Institute of Natural Resources, Chicago.

Bagnold, R. A., 1965: The Physics of Blown Sand and Desert Dunes. Methuen and Co., Ltd., London, 265 pp.

Bisal, F., and K. F. Nielsen, 1962: Movement of soil particles in saltation. Canadian J. Soil Sci., $\underline{42}, 81-86$.

Bender, G. M., 1982. Personal communication. Mr. Bender is associated with the Water Survey's Nationwide Urban Runoff Program.

Brown, M. B., Editor, 1977: BMDP-77 Biomedical Computer Programs, P-Series, University of California Press, Berkeley, 880 pp.

Carlson, T. N., and J. M. Prospero, 1972: The large-scale movement of Saharan air outbreaks over the northern equitorial Atlantic. J. App1. Meteoro1., 11, 283-297.

Chepil, W. S., 1944: Utilization of crop residues for wind erosion contro1. Sci. Agric., 26, 307-319.

Chepil, W. S., 1945a: Dynamics of wind erosion: I. Nature of movement of soil by wind. Soil Sci., $60,305-320$.

Chepil, W. S., 1945b: Dynamics of wind erosion: II. Initiation of soil 
movement. Soil Sci., $\underline{60}, 397-411$.

Chepil, W. S., 1945c: Dynamics of wind erosion: III. The transport capacity of the wind. Soil Sci., $60,475-480$.

Chepil, W. S., 1946a: Dynamics of wind erosion: V. Cumulative intensity of soil drifting across eroding fields. Soil Sci., 61, 257-263.

Chepil, W. S., 1946b: Dynamics of wind erosion: VI. Sorting of soil material by the wind. Soil Sci., $\underline{61}, 331-340$.

Chepil, w. S., 1956: Influence of moisture on erodibility of soil by wind. Soil Sci. Soc. Am. Proc., 20, 288-292.

Chepil, W. S., 1957a: Sedimentary characteristics of dust storms: I. Sorting of wind-eroded soil material. Am. J. Sci., 225, 12-22. Chepil, W. S., 1957b: Sedimentary characteristics of dust storms: III. Composition of suspended dust. Am. J. Sci., 255, 206-213.

Chepil, W. S., 1959: Equilibrium of soil grains at the threshold of movement by wind. Soil Sci. Soc. Am. Proc., 23, 422-428.

Chepil, W. S., and R. A. Milne, 1941: Wind erosion in soil in relation to roughness of surface. Soil Sci., 52, 417-433.

Chepil, W. S.,, F. H. Siddoway, and D. V. Armbrust, 1962: Climatic factor for estimating wind erodibility of farm fields. J. Soil Water Conserv., 17, 162-165.

Chepil, W. S., and N. P. Woodruff, 1963: The physics of wind erosion and its control. Adv. Agron., 15, 211-302.

Chepil, W. S., N. P. Woodruff, F. H. Siddoway, D. W. Fryrear, and D. V. Armbrust, 1963: Vegetative and nonvegetative materials to control wind and water erosion. Soil Sci. Soc. Am. Proc., 27, 86-89. Chepil, W. S., N. P. Woodruff, and A. W. Zingg, 1955: Field study of 
wind erosion in western Texas. Report SCS-TP-125, U.S. Department of Agriculture, Washington, D.C.

Clayton, R. N., R. W. Rex, J. K. Syers, and M.L. Jackson, 1972: Oxygen isotope abundance in quartz from Pacific pelagic sediments. J. Geophys. Res., 11, 3907-3915.

Countess, R. J., G. T. Wolff, and S. H. Cadle, 1980: The Denver winter aerosol: a comprehensive chemical characterization. J. Air Pollut. Contro1 Assoc., 30, 1194-1200.

Cowherd, C., and P. Englehart, 1982: Characterization of fine particulate emission factors for paved roads. Paper presented at the Fifth Symposium on Fugitive Emissions: Measurement and Contro1, May 3-5, Charleston, SC.

Cowherd, C., C. M. Guenther, and D. D. Wallace, 1974: Emission inventory of agricultural tilling, unpaved roads and airstrips, and construction sites. Report EPA 450/3-74-085, U.S. Environmental Protection Agency, Research Triangle Park, NC.

Cowherd, C., and C. 0. Mann, 1976: Quantification of dust entrainment from paved roads. Paper presented at the 69th Annual Meeting of the APCA, Portland, OR.

Craig, D. G., and J. W. Tupelle, 1964: Guide for wind erosion control on cropland in the Great Plains states. Soil Conservation Service, U.S. Department of Agriculture, Washington, D.C.

Daniel, H. A., 1936: Physical changes in soils of the Southern High Plains due to cropping and wind erosion and the relation between the (sand and silt)/clay ratios in these soils. J. Am. Soc. Agron., 28, $570-580$. 
Dzubay, T. G., 1980: Chemical element balance method applied to dichotomous sampler data. Ann. New York Acad. Sci., 338, 126-144. Englehorn, C. L., A. W. Zingg, and N. P. Woodruff, 1952: The effects of plant residue cover and clod structure on soil losses by wind. Soil Sci. Soc. Am. Proc., 16, 29-33.

Dzubay, T. G., R. K. Stevens, W. J. Courtney, and E. A. Drane, 1981:

Chemical element balance analysis of Denver aerosol. In: Russell, P. A., Ed., Electron Microscopy and X-Ray Applications to Environmental and Occupational Health Analysis, Vol. II. Ann Arbor Science, Ann Arbor, MI, pp. 23-42.

Evans, J. S., and D. W. Cooper, 1980: An inventory of particulate emissions from open sources. J. Air Pollut. Control Assoc., 30, 1298-1303.

Evans, J. S., and D. W. Cooper, 1981: The contributions of open sources to ambient TSP levels. J. Air Pollut. Control Assoc., 31, 549-556.

Fisher, P. S., and E. L. Skidmore, 1970: A Fortran IV program to solve the wind erosion equation. Report ARS 41-174, U.S. Department of Agriculture, Washington, D.C.

Free, E. E., 1911: The movement of soil material by the wind. USDA Bulletin 68, 271 pp. U.S. Department of Agriculture, Washington, D.C.

Fryrear, D. W., and L. Lyles, 1977: Wind erosion research accomplishments and needs. Trans. ASAE, 20, 916-918.

Gaarenstroom, P. D., S. P. Perone, and J. L. Moyers, 1977: Application of pattern recognition and factor analysis for characterization of atmospheric particulate composition in southwest desert atmosphere. 
Environ. Sci. Techno1., 11, 795-800.

Gatz, D. F., 1975: Relative contributions of different sources of urban aerosols: application of a new estimation method to multiple sites in Chicago. Atmos, Environ., 9, 1-18.

Gatz, D. F., 1978: Identification of aerosol sources in the St. Louis area using factor analysis. J. Appl. Meteorol., 17, 600-608.

Gillette, D. A., 1974: On the production of soil wind erosion aerosols having the potential for long-range transport. J. Rech. Atmos., $\underline{8}$, 735-744.

Gillette, D. A., I. H. Blifford, and C. R. Fenster, 1972: Measurements of aerosol size distribution and vertical fluxes of aerosols on land subject to wind erosion. J. App1. Meteoro1., 11, 977-987.

Gordon, G. E., 1980a: Techniques for treating multielement particulate data to obtain information on sources: an overview. Ann. New York Acad. Sci., 338, 93-102.

Gordon, G. E., 1980b: Receptor models. Environ. Sci. Techno1., 14, 792-800.

Hagen, L. J., and N. P. Woodruff, 1973: Air pollution from dust storms in the Great Plains. Atmos, Environ., I, 323-332.

Hayes, W. A., 1972: Designing wind erosion control systems in the Midwest region. Technical Note, Agronomy, LI-9, Soil Conservation Service Regional Technical Service Center, Lincoln, NE, 23 pp. Hayes, W. A., 1965: Wind erosion equation useful in designing northeastern crop protection. J. Soil Water Conserv., 20, 153-155. Hayes, W. A., 1966: Guide for wind erosion control in the northeastern states. Soil Conservation Service, U.S. Dept. of Agriculture, Opper 
Darby, PA.

Hidy, G. M., and J. R. Brock, 1970: An assessment of the global sources of tropospheric aerosols. Proc., Second Clean Air Congress, Washington, D.C., December, 1970.

Hopke, P. K., E. S. Gladney, G. E. Gordon, W. H. Zoller, and A. G. Jones, 1976: The use of multivariate analysis to identify sources of selected elements in the Boston urban aerosol. Atmos. Environ., 10, $1015-1025$.

Judson, S., 1968: Erosion of the land--or what's happening to our continents? Am. Sci., $\underline{56}, 356-374$.

Kolak, N. P., and J. R. Visalli, 1981: Comparison of three methods for measuring suspended-particulate concentrations. Environ. Sci. Technol.,. 15, 219-223.

Kowalczyk, G. S., G. B. Gordon, and S. W. Rheingrover, 1982: Identification of atmospheric particulate sources in Washington, D.C., using chemical element balances. Environ. Sci. Technol., 16, 79-90.

Leaderer, B. P., 1978: Summary of the New York Summer Aerosol Study (NYSAS). J. Air Pollut. Control Assoc., 28, 321-327.

Lyles, L., 1975: Possible effects of wind erosion on soil productivity. J. Soil Water Conserv., 30, 279-283.

Lyles, L., and R. K. Krauss, 1971: Threshold velocities and initial particle motion as influenced by air turbulence. Trans. ASAE, 14, $563-566$.

Lyles, L., R. L. Schrandt, and N. F. Schmiedler, 1974: How aerodynamic roughness elements control sand movement. Irans. ASAE, 17, 134-139. 
Lynn, D. A., G. I. Deane, R. C. Galkiewicz, and R. M. Bradway, 1976: National assessment of the urban particulate problem. Vol. I -Summary of National Assessment. U.S. Environmental Protection Agency, EPA-450/3-76-024, Research Triangle Park, NC.

Malina, F. J., 1941: Recent developments in the dynamics of wind erosion. Trans. Am. Geophys. Union, 22, 262-284.

Moldenhauer, W. C., and E. R. Duncan, 1969: Principles and methods of wind erosion control in Iowa. Special Report 62, Agriculture and Home Economics Experiment Station, Iowa State University, Ames 50010 .

Moss, H. C., 1935: Some field and laboratory studies of soil drifting in Saskatchewan. Sci. Agric., 15, 665-675.

Newman, J. E.,'M. D. Abel, P. R. Harrison, and K. J. Yost, 1976: Wind as related to critical flushing speed by high-volume sampler particulate loading. In, Engelmann, R. J., and G. A. Sehmel, Coordinators, Atmosphere-Surface Exchange of Particulate and Gaseous Pollutants (1974), PP. 466-495.

Niles, J. S., 1961: A universal equation for measuring wind erosion. ARS Special Report 22-69, Agricultural Research Service, D.S. Department of Agriculture, Washington, D.C.

Ortiz, C. A., 1978: Aerosol collection characteristics of ambient aerosol samplers. M.S. Thesis, Texas A\& M University, College Station, Texas.

Patterson, E. M., D. A. Gillette, and G. W. Grams, 1976: The relation between visibility and the size-number distribution of airborne soil particles. J. Appl. Meteorol., 15, 470-478. 
Rahn, K. A., R. D. Borys, G. E. Shaw, L. Schutz, and R. Jaenicke, 1979: Long-range impact of desert aerosol on atmospheric chemistry: two examples. In: Morales, C., Ed., Saharan Dust, SCOPE 14, John Wiley, New York, 297 pp.

Siddoway, F. H., W. S. Chepil, and D. V. Armbrust, 1965: Effect of kind, amount, and placement of residue on wind erosion control. Trans. $\underline{\text { ASAE }}, \underline{8}, 327-331$.

Skidmore, E. L., 1965: Assessing wind erosion forces: direction anc relative magnitudes. Soil Sci. Soc. Am. Proc., 29, 587-590.

Skidmore, E. L., P. S. Fisher, and N. P. Woodruff, 1970: Wind erosion equation: computer solution and application. Soil Sci. Soc. Am. Proc., 34, 931-935.

Skidmore, E. L., N. L. Nossaman, and N. P. Woodruff, 1966: Wind erosion as influenced by row spacing, row direction, and grain sorghum population. Soil Sci. Soc. Am. Proc., 39, 505-509.

Skidmore, E. L., and N. P. Woodruff, 1968: Wind erosion forces in the United States and their use in predicting soil loss. Agriculture Handbook No. 346, Agricultural Research Service, O.S. Department of Agriculture, Washington, D.C.

Smith, S. J., D. J. Fingleton, and T.-L. 0. Gedo, 1981: A review of concentrations, chemical composition, size distribution and health effects related to inorganic inhalable particulates in Illinois. Document No. $81 / 13$, Illinois Institute of Natural Resources, Chicago.

Terstriep, M. L., G. M. Bender, and D. C. Noel, 1981: National Urban Runoff Project, Champaign, Illinois. Evaluation of the 
Effectiveness of Municipal Street Sweeping in the Control of Urban Storm Runoff Pollution. First Annual Report, Prepared for the Illinois Environmental Protection Agency and the U. S. Environmental Protection Agency, Region V. Illinois State Water Survey, Champaign.

Spengler, J. D., W. A. Turner, F. P. Fairchild, J. E. Slaughter, and T. G. Dzubay, 1981: Operation manual for automatic dichotomous samplers: application to Beckman dichotomous samplers. Report EPA 600/8-81-007. Harvard School of Public Health. Environmental Sciences Research Laboratory, U.S. EPA, Research Triangle Park, NC 27711 .

Wilson, L., 1975: Application of the wind erosion equation to predict fugitive dust emissions. J. Soil Water Conserv., 30, 215-219. Woodruff, N. P., and D. V. Armbrust, 1968: A monthly climatic factor for the wind erosion equation. J. Soil Water Conserv., 23, 103-104. Woodruff, N. P., L. Lyles, F. H. Siddoway, and D. W. Fryrear, 1977: How to control wind erosion. Agricultural Information Bulletin No. 354, U.S. Department of Agriculture, Washington, D.C.

Woodruff, N. P., and F. H. Siddoway, 1965: A wind erosion equation. Soil Sci. Soc. Am. Proc., 29, 602-608.

U.N.F.A.O., 1960: Soil erosion by wind and measures for its contol on agricultural lands. Agricultural Development Paper No. 71, United Nations Food and Agriculture Organization, Rome.

Zingg, A. W., 1954: The wind erosion problem in the Great Plains. Trans. Am. Geophys. Union, 35, 252-258. 
Characterization of Urban and Rural Inhalabie Particulates

thor $(x)$

Donald F. Gatz, Susan T. Wiley, Lih-Ching Chu Morming Orzanlzution Name and Nodreas

IL Department of Energy and Natural Resources

State Water Survey Division

605 East Springfield Avenue

P. 0. Box 5050, Station A

Champaign, IL

I1linois Department of Energy and Natural Resources 325 W. Adams Street

Springfield, IL 62706

he Clean Air Act emphasizes that health aspects should be considered very strongly when ssessing effects of air pollutants. This has caused a concern at the Federal level that he standard high volume (hivol) sampler does not provide a proper sample of airborne articles for use in assessing health effects.

$n$ order to be prepared for potential future Federal requirements in the area of TSP ampling, the IEPA needs comparative measurements or airborne particles using the tandard hivol and the various samplers such as the two stage dichotomous sampler. This eport compares concentrations of inhalable and total airborne mass in both urban and rural reas, looks at the effects of street sweeping on urban air quality, by comparing hivol and ichotomous sampler measurements in urban areas in the presence and absence of regular treet sweeping and determines the sources if airborne particulate matter, and their elative contributions to TSP in an urban area.

lichotomous Sampler livol Sampler

\section{- cosati neld/Group}

ivellobility stutemen: No restriction on distribution. Ivailable at the IL depository libraries or from the National Technical Information Service, ipringfield. VA 22161

\begin{tabular}{|c|c|}
\hline $\begin{array}{l}\text { 19. Securto Cless (This Aeports } \\
\text { Unclassified }\end{array}$ & $\begin{array}{c}\text { 21. Na. of Pagen } \\
108 \\
\end{array}$ \\
\hline $\begin{array}{l}\text { 20. Socurity Clos: This Pores) } \\
\text { Unclassified }\end{array}$ & 2 Pric \\
\hline
\end{tabular}






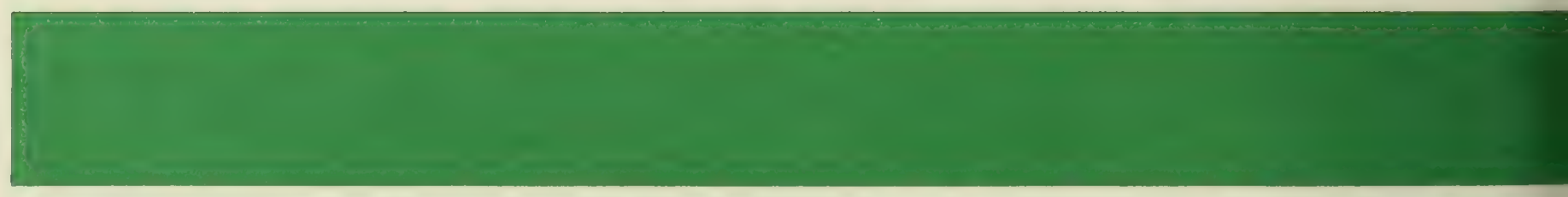

F 


UNIVEASITY OF ILLINOIS-URBANA

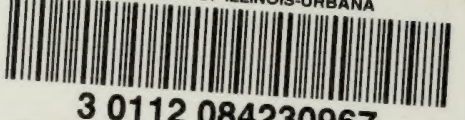

30112084230967 